\title{
Finite deformation of single-walled carbon nanocones under axial compression using a temperature-related multiscale quasi-continuum model
}

\author{
Xiangyang Wang a, , Jinbao Wang b, Xu Guo c,* \\ a School of Transportation, Ludong University, Yantai, Shandong, China, 264025 \\ ${ }^{b}$ School of Shipping and Ports Architecture Engineering, Zhejiang Ocean University, China, 316022 \\ c State Key Laboratory of Structural Analysis for Industrial Equipment, \\ Department of Engineering Mechanics, Dalian University of Technology, China, 116023
}

\begin{abstract}
A temperature-related multiscale quasi-continuum (QC) model is presented herein for the investigation of the finite deformation behaviors, especially buckling and post-buckling, of open-tip single-walled carbon nanocones (SWCNCs) in thermal environments. The hyper-elastic constitutive model is established with the use of so-called temperature-related higher-order Cauchy-Born (THCB) rule as the kinematic description for the deformation of SWCNCs. The corresponding meshless computational scheme is developed to accomplish the numerical simulation of the deformation responses of SWCNCs. The numerical results reveal that the present approach is effective and efficient for the prediction of the buckling behaviors of SWCNCs at finite temperature. It is found that the buckling and post-buckling of SWCNCs are strongly dependent on the
\end{abstract}

\footnotetext{
* Corresponding authors.

Xiangyang Wang Tel: +86-535-6696123. E-mail addresses: wxy017@126.com

Xu Guo Tel: +86-411-84707807. E-mail addresses: guoxu@dlut.edu.cn 
rotation angle of cutting lines, the top radius, the height and the apex angle. The mechanical properties and buckling of SWCNCs with large top radius and apex angle are close to those of graphene.

Keywords: Single-walled carbon nanocones; Multiscale quasi-continuum model; Temperature-related higher-order Cauchy-Born rule; Meshless method; Buckling. 


\section{Introduction}

Similar to carbon nanotube (CNT), carbon nanocone (CNC) as one of synthesized allotropes of carbon also has unique mechanical and physical properties. Nowadays, much more researchers have been paying close attention towards CNCs. Ansari et al. [1] reported that decreasing apex angle and the length of $\mathrm{CNC}$ results in an increase in the natural frequency via molecular mechanics (MM) method and molecular dynamics (MD) simulations. Fakhrabadi et al. [2] adopted MM approach to investigate the elastic and buckling properties of CNCs with different apex angles and lengths. Based on MD simulation, Jordan and Crespi [3] studied the chiral inversion behaviors of closed SWCNCs subjected to compression. With use of MD simulations, Liew et al. [4] analyzed buckling behaviors of SWCNCs under compression. They revealed that for a fixed height/bottom radius ratio, SWCNCs with smaller top radii are much stiffer.

Except for molecular based approaches, some continuum and quasi-continuum (QC) models have been proposed for CNCs successively. Lee and Lee [5] employed finite element method to study the vibrational behaviors of fixed beam and cantilever SWCNCs. They found that the natural frequencies of SWCNCs increased significantly at higher vibrational modes. Wei and Srivastava [6] developed a continuum model to study the Young's modulus of SWCNCs which is $\cos ^{4} \theta$ of that of an equivalent single-walled carbon nanotube (SWCNT). Yan et al. [7-10] employed higher-order Cauchy Born (HCB) rule to form a QC based mesh-free computational scheme to study the mechanical properties and buckling of nanotubes and nanocones under different boundary conditions, and the numerical results agree well with MD simulations. Ansari et al. [11-12] studied the 
mechanical properties and vibrational behaviors of CNCs with use of spring-mass model and shell model, respectively. Furthermore, the same research team has taken the van der waals interaction into consideration to investigate the contact behaviors of CNCs with other nanomaterials [13-15]. Firouz-Abadi et al. [16] proposed a nonlocal continuum shell model for the analysis of the stability of nanocones. Based on the developed continuum model, Mustapha and Zhong [17] investigated the buckling of SWCNCs and reported that the apex angle has an increasing effect on critical axial compressive load.

Generally, CNCs and CNCs based nanostructures often work in thermal environments. In order to have a thorough knowledge of mechanical responses of CNCs, the temperature effect should be taken into consideration. However, to the best of the author's knowledge, few of studies handle this issue. Tsai and Fang [18] adopted MD simulations to study the thermal stability of CNCs. The simulation results showed that the melting temperature of CNCs increases with cone apex angle increasing. Li et al. [19] predicted the mechanical properties of SWCNCs using MD simulations. They found that the ultimate extension loading decreases with the temperature increasing. Liao et al. [20] investigated the influence of temperature on the deformation behaviors of open-tip CNCs subjected to tensile and compressive loadings. It is shown that the tensile failure strain and the compressive critical strain decline evidently with the system temperature increasing. Obviously, the temperature is one of the important influence factors of mechanical responses of CNCs.

The central idea of the present paper is to develop a highly effective and efficient numerical simulation approach to accurately predict the mechanical properties and 
buckling of SWCNCs at finite temperature, which can overcome the limitation of molecular based approaches with small atomistic systems and time consuming. A temperature-related multi-scale QC model is constructed herein for SWCNCs based on the temperature-related higher-order Cauchy-Born (THCB) rule which was proposed by Guo et al. [21] for the analysis of thermo-mechanical properties of CNTs. The effectiveness of THCB rule has been confirmed by the successfully application on the investigation of graphene and SWCNTs [22-24]. The meshless method [25] which can naturally meet the requirement of the high order continuity of the proposed QC model is employed to construct the meshless numerical computational scheme for investigation of the buckling and post-buckling of SWCNCs at finite temperature. The prominent characteristics of the present approach are that some important microstructure information, such as chirality, of SWCNCs can be described accurately and the computational efforts can be saved greatly compared with MD simulations.

\section{Temperature-related multiscale quasi-continuum constitutive model}

In this section, the temperature-related multiscale quasi-continuum (QC) constitutive model based on the temperature-related higher-order Cauchy-Born (THCB) rule [21] is proposed for the study of the mechanical responses of single-walled carbon nanocones (SWCNCs).

\subsection{THCB rule}

For the analysis of the thermo-mechanical properties and buckling of single-walled 
carbon nanotubes (SWCNTs) at finite temperature, Guo et al. [21] proposed a smooth virtual hexagonal carbon lattice structure model formed by vibration centers of carbon atoms at non-zero temperature and simultaneously presented the so-called THCB rule. The THCB rule can be employed to describe the kinematic relationships of deformation of thin-walled nanocrystalline with one atom thickness and provide a linkage between the microscopic and macroscopic level at finite temperature. It has been successfully used to study the thermo-mechanical properties and buckling behaviors of SWCNTs and graphene [22-24], and the numerical results are in good agreement with molecular dynamic (MD) simulations. In the THCB rule, the deformation of lattice vector can be described as

$$
\boldsymbol{r}_{I J}(T)=\boldsymbol{F} \cdot \boldsymbol{R}_{I J}(T)+\frac{1}{2} \boldsymbol{G}:\left(\boldsymbol{R}_{I J}(T) \otimes \boldsymbol{R}_{I J}(T)\right),
$$

where $\boldsymbol{R}_{I J}(T)$ and $\boldsymbol{r}_{I J}(T)$ are the bond vectors between the vibration centers of atoms $I$ and $J$ in the initial reference configration and the current counterpart at the prescribed temperature $T$, respectively. $\boldsymbol{F}$ and $\boldsymbol{G}$ denote first- and second-order deformation gradient tensors, respectively. It is worth noting that since the hexagonal lattice structure of SWCNCs is non-centro symmetric, an inner relaxation displacement should be added to maintain the internal equilibrium of the atomistic system [21-24], and then the corresponding THCB rule for SWCNCs should be rewritten as

$$
\boldsymbol{r}_{I J}(T)=\boldsymbol{F} \cdot\left(\boldsymbol{R}_{I J}(T)+\boldsymbol{\xi}_{I}(T)\right)+\frac{1}{2} \boldsymbol{G}:\left[\left(\boldsymbol{R}_{I J}(T)+\boldsymbol{\xi}_{I}(T)\right) \otimes\left(\boldsymbol{R}_{I J}(T)+\boldsymbol{\xi}_{I}(T)\right)\right],
$$

where $\xi_{I}(T)=\xi_{I}(T)\left(\xi_{I 1}(T), \xi_{I 2}(T)\right)^{T}$ represents the inner relaxation vector of the vibration center of atom $I$, which can be determined by energy minimization of the representative cell. 
Generally, SWCNC is often viewed as being formed by rolling up a planar fan-shaped graphene sheet into a conical shape seamlessly. A fan-shaped graphene sheet can be 'tailored' as shown in Fig. 1. The angles between the cutting lines $O A$ and $O B$ can be only taken five discrete values $60^{\circ}, 120^{\circ}, 180^{\circ}, 240^{\circ}$ and $300^{\circ}$ which are corresponding to the conical angles of SWCNCs $19.2^{\circ}, 38.9^{\circ}, 60^{\circ}, 84.6^{\circ}$ and $112.9^{\circ}$, respectively [26]. In the present study, the rotation angle of the cutting lines around point $O$ is defined as $\theta_{r}$ and $\theta_{r}>0$ when the rotation is anticlockwise (the included angle between the two cutting lines remain unchanged). $\theta_{r}$ of the cutting lines depict in Fig. 1 is prescribed as $0^{\circ}$. It can be observed clearly from Fig. 1 that the chirality of SWCNC is determined by the value of $\theta_{r}$. The dependency of $\theta_{r}$ on the mechanical responses of SWCNCs will be studied in the following section.

The rolling process is, however, by no means a pure geometry transformation. The carbon nanocone will relax itself to attain a state with minimum energy. In order to allow for this relaxation, two parameters $\lambda_{1}(T)$ and $\lambda_{2}(T)$ defined on the initial configuration (i.e., the planar virtual fan-shaped graphene sheet formed by vibration centers of all the carbon atoms) along the radial and circumferential directions, respectively, are introduced to describe the configuration of the nanocone after relaxation. It should be noted that the relaxation parameters are not on the current configuration. The transformation mapping from the planar virtual fan-shaped graphene sheet to the undeformed SWCNC can be established as (see Fig. 2 for reference) 


$$
\left\{\begin{array}{c}
x_{1}=\frac{\lambda_{1}(T) \sqrt{X_{1}^{2}+X_{2}^{2}} \sqrt{4 \pi^{2}-\lambda_{2}^{2} \theta_{g}^{2}}}{2 \pi} \\
x_{2}=\frac{\lambda_{1}(T) \lambda_{2}(T) \theta_{\mathrm{g}}}{2 \pi} \sqrt{X_{1}^{2}+X_{2}^{2}} \cos \left(2 \pi \frac{\phi}{\theta_{g}}\right) \\
x_{3}=\frac{\lambda_{1}(T) \lambda_{2}(T) \theta_{\mathrm{g}}}{2 \pi} \sqrt{X_{1}^{2}+X_{2}^{2}} \sin \left(2 \pi \frac{\phi}{\theta_{g}}\right)
\end{array}\right.
$$

where $\boldsymbol{X}=\left(X_{1}, X_{2}\right)^{T}$ is the coordinate vector of a point in the configuration of the fan-shaped graphene sheet and $\boldsymbol{x}=\left(x_{1}, x_{2}, x_{3}\right)^{T}$ is the image of $\boldsymbol{X}$ in the configuration of the undeformed SWCNC. $\theta_{g}$ denotes the angle of the tailored graphene sector. $\phi=\operatorname{actan}\left(X_{2} / X_{1}\right)$. The relaxation parameters $\lambda_{1}(T)$ and $\lambda_{2}(T)$ can be determined through an energy minimization process of free energy density.

Based on Eq. (3), the transformation mapping between the deformed SWCNC and the planar fan-shaped graphene sheet can be established as

$$
\left\{\begin{array}{c}
x_{1}=\frac{\lambda_{1}(T) \sqrt{X_{1}^{2}+X_{2}^{2}} \sqrt{4 \pi^{2}-\lambda_{2}^{2} \theta_{g}^{2}}}{2 \pi}+u_{1}\left(X_{1}, X_{2}\right) \\
x_{2}=\frac{\lambda_{1}(T) \lambda_{2}(T) \theta_{\mathrm{g}}}{2 \pi} \sqrt{X_{1}^{2}+X_{2}^{2}} \cos \left(2 \pi \frac{\phi}{\theta_{g}}\right)+u_{2}\left(X_{1}, X_{2}\right) \\
x_{3}=\frac{\lambda_{1}(T) \lambda_{2}(T) \theta_{\mathrm{g}}}{2 \pi} \sqrt{X_{1}^{2}+X_{2}^{2}} \sin \left(2 \pi \frac{\phi}{\theta_{g}}\right)+u_{3}\left(X_{1}, X_{2}\right)
\end{array}\right.
$$

where the displacement vector $\boldsymbol{u}=\left(u_{1}, u_{2}, u_{3}\right)^{T}$ describe the deformation between the undeformed SWCNC and deformed counterpart. The deformation gradient tensor $\boldsymbol{F}$ and $\boldsymbol{G}$ in Eq. (2) can be calculated with use of Eq. (4) as

$$
\boldsymbol{F}=\frac{\partial \boldsymbol{x}}{\partial \boldsymbol{X}}, \quad \boldsymbol{G}=\frac{\partial^{2} \boldsymbol{x}}{\partial \boldsymbol{X} \otimes \partial \boldsymbol{X}}
$$

\subsection{Temperature-related multiscale quasi-continuum constitutive model}

In the present study, Helmholtz free energy is employed to describe the 
thermodynamic potential of SWCNCs, which has been successfully utilized for the analysis of carbon based nano-materials [21-24]. With use of the quasi harmonic approximation and the local harmonic approximation, the couplings between the vibrations of different carbon atoms can be totally neglected reasonably [21,27]. Under this circumstance, the Helmholtz free energy $A$ of an atom system can be simplified as

$$
A=U_{0}+N k_{B} T \sum_{\alpha=1}^{3} \ln \left(2 \sinh \left(\frac{\hbar \omega_{\alpha}}{2 k_{B} T}\right)\right),
$$

where $U_{0}$ denotes the total potential energy of the considered SWCNC and $N$ represents the total number of carbon atoms. $k_{B}=1.38 \times 10^{-23} \mathrm{~J} \mathrm{~K}^{-1}$ and $\hbar=1.05 \times 10^{-34} \mathrm{~J} \mathrm{~s}$ are the Boltzmann constant and the Planck's constant, respectively. $\omega_{\alpha}(\alpha=1,2,3)$ is the $\alpha-$ th order normal vibration frequency of one atom, which can be determined by

$$
\left|\frac{1}{m_{C}} \frac{\partial^{2} U_{0}}{\partial \boldsymbol{x}_{I} \otimes \partial \boldsymbol{x}_{I}}\right|_{x_{I}=x_{I}^{0}}-\boldsymbol{I}_{3 \times 3}\left(\omega_{I}\right)^{2} \mid=0,
$$

where $m_{C}$ denotes the mass of carbon atom. $\partial^{2} U_{0} / \partial \boldsymbol{x}_{I} \otimes \partial \boldsymbol{x}_{I}$ is the local dynamic matrix with the assumption that only the considered atom $I$ vibrates and all the surrounding atoms are fixed. $\boldsymbol{x}_{I}^{0}$ represents the vibration center of atom $I . \boldsymbol{I}_{3 \times 3}$ is the $3 \times 3$ identity matrix.

The surface free energy density can be obtained through the homogenization of Helmholtz free energy as

$$
W_{T}\left(\boldsymbol{r}_{I J}(T)\right)=\frac{U_{0 I}\left(\boldsymbol{r}_{I J}(T)\right)+\Psi_{T I}\left(\boldsymbol{r}_{I J}(T)\right)}{\Omega_{I}}
$$

where $\Omega_{I}$ is the average area of a carbon atom in the reference configuration which can be calculated as $3 \sqrt{3} a_{0}^{2} / 4$, the representative atom and the corresponding unit cell are shown in Fig. 3 where $a_{0}$ is the equilibrium bond length which can be calculated through 
an energy minimization process. The variation of the equilibrium bond length with respect to temperature is depicted in Fig. 4.

$$
\Psi_{T I}\left(\boldsymbol{r}_{I J}(T)\right)=k_{B} T \sum_{\alpha=1}^{3} \ln \left(2 \sinh \left(\frac{\hbar \omega_{I \alpha}\left(\boldsymbol{r}_{I J}(T)\right)}{2 k_{B} T}\right)\right),
$$

and $U_{0 I}$ is the potential energy of atom $I$, described as

$$
U_{0 I}\left(\boldsymbol{r}_{I J}(T)\right)=\frac{\sum_{J=1}^{3} V\left(\boldsymbol{r}_{I J}(T)\right)}{2}
$$

where $V$ denotes potential energy which can be calculated by Tersoff-Brenner potential $[28,29]$,

$$
V\left(r_{I J}\right)=V_{R}\left(r_{I J}\right)-\bar{B}_{I J} V_{A}\left(r_{I J}\right)
$$

where $r_{I J}=\left|\boldsymbol{r}_{I J}\right|$ denotes the distance between atoms $I$ and $J$. The repulsive energy $V_{R}$, the attractive energy $V_{A}$ and the multi-body coupling effect $\bar{B}_{I J}$ have the following forms, respectively

$$
\begin{gathered}
V_{R}(r)=\frac{D^{(e)}}{S-1} e^{-\sqrt{2 S} \beta\left[r-R^{(e)}\right]} f_{c}(r), \\
V_{A}(r)=\frac{D^{(e)}}{S-1} S e^{-\sqrt{2 / S} \beta\left[r-R^{(e)}\right]} f_{c}(r), \\
\bar{B}_{I J}=\frac{1}{2}\left(B_{I J}+B_{J I}\right),
\end{gathered}
$$

with

$$
\begin{gathered}
B_{I J}=\left[1+\sum_{K(\neq I, J)} G\left(\theta_{I J K}\right) f_{c}\left(r_{I K}\right)\right]^{-1 / 2}, \\
f_{c}(r)=\left\{\begin{array}{cc}
1 & 1 \\
\left.\frac{1}{2} 1+\cos \left(\frac{\pi\left(r-R^{(1)}\right)}{R^{(2)}-R^{(1)}}\right)\right\} & R^{(1)}<r \leq R^{(2)}, \\
0 & r>R^{(2)}
\end{array}\right. \\
G(\theta)=a_{0}\left[1+\frac{c_{0}^{2}}{d_{0}^{2}}-\frac{c_{0}^{2}}{d_{0}^{2}+(1+\cos \theta)^{2}}\right],
\end{gathered}
$$

where the parameters are taken as $D^{(e)}=6.000 \mathrm{eV}, S=1.22, \beta=21 \mathrm{~nm}^{-1}, \quad R^{(e)}=$ 
$0.1390 \mathrm{~nm}, R^{(1)}=0.17 \mathrm{~nm}, R^{(2)}=0.20 \mathrm{~nm}, a_{0}=0.00020813, c_{0}=330, d_{0}=3.5$.

As mentioned in the previous section, the relaxation parameters can be obtained through minimizing the surface free energy density as

$$
\frac{\partial W_{T}}{\partial \lambda_{1}(T)}=\frac{\partial W_{T}}{\partial \lambda_{2}(T)}=0
$$

The first Piola-Kirchhoff stress tensor $\boldsymbol{P}_{T}$ and the higher-order stress tensor $\boldsymbol{Q}_{T}$ can be derived from Eq. (8) as

$$
\begin{aligned}
& \boldsymbol{P}_{T}=\frac{\partial W_{T}}{\partial \boldsymbol{F}}=\left.\frac{1}{\Omega_{I}}\left(\frac{\partial U_{0 I}}{\partial \boldsymbol{F}}+\frac{\hbar}{2} \sum_{\alpha=1}^{3} \operatorname{coth}\left[\frac{\hbar \omega_{I \alpha}}{2 k_{B} T}\right] \frac{\partial \omega_{I \alpha}}{\partial \boldsymbol{F}}\right)\right|_{\zeta_{I}=\check{\xi}_{I}}, \\
& \boldsymbol{Q}_{T}=\frac{\partial W_{T}}{\partial \boldsymbol{G}}=\left.\frac{1}{\Omega_{I}}\left(\frac{\partial U_{0 I}}{\partial \boldsymbol{G}}+\frac{\hbar}{2} \sum_{\alpha=1}^{3} \operatorname{coth}\left[\frac{\hbar \omega_{I \alpha}}{2 k_{B} T}\right] \frac{\partial \omega_{I \alpha}}{\partial \boldsymbol{G}}\right)\right|_{\zeta_{I}=\check{\xi}_{I}},
\end{aligned}
$$

where $\breve{\xi}_{I}$ is the equilibrium solution of $\xi_{I}$, which can be determined by

$$
\frac{\partial W_{T}}{\partial \xi_{I}}=0
$$

The tangent moduli can be obtained as follows

$$
\begin{aligned}
\boldsymbol{M}_{\boldsymbol{F F}}=\frac{\partial \boldsymbol{P}_{T}}{\partial \boldsymbol{F}}= & \frac{1}{\Omega_{I}}\left[\left(\frac{\partial^{2} U_{0 I}}{\partial \boldsymbol{F} \otimes \partial \boldsymbol{F}}+\frac{\partial^{2} \Psi_{T I}}{\partial \boldsymbol{F} \otimes \partial \boldsymbol{F}}\right)-\left(\frac{\partial^{2} U_{0 I}}{\partial \boldsymbol{F} \otimes \partial \xi_{I}}+\frac{\partial^{2} \Psi_{T I}}{\partial \boldsymbol{F} \otimes \partial \xi_{I}}\right)\right. \\
& \left.\cdot\left(\frac{\partial^{2} U_{0 I}}{\partial \xi_{I} \otimes \partial \xi_{I}}+\frac{\partial^{2} \Psi_{T I}}{\partial \xi_{I} \otimes \partial \xi_{I}}\right)^{-1} \cdot\left(\frac{\partial^{2} U_{0 I}}{\partial \xi_{I} \otimes \partial \boldsymbol{F}}+\frac{\partial^{2} \Psi_{T I}}{\partial \xi_{I} \otimes \partial \boldsymbol{F}}\right)\right]\left.\right|_{\xi_{I}=\check{\xi}_{I}}, \\
\boldsymbol{M}_{\boldsymbol{F G}}=\frac{\partial \boldsymbol{P}_{T}}{\partial \boldsymbol{G}}= & \frac{1}{\Omega_{I}}\left[\left(\frac{\partial^{2} U_{0 I}}{\partial \boldsymbol{F} \otimes \partial \boldsymbol{G}}+\frac{\partial^{2} \Psi_{T I}}{\partial \boldsymbol{F} \otimes \partial \boldsymbol{G}}\right)-\left(\frac{\partial^{2} U_{0 I}}{\partial \boldsymbol{F} \otimes \partial \xi_{I}}+\frac{\partial^{2} \Psi_{T I}}{\partial \boldsymbol{F} \otimes \partial \xi_{I}}\right)\right. \\
& \left.\cdot\left(\frac{\partial^{2} U_{0 I}}{\partial \xi_{I} \otimes \partial \xi_{I}}+\frac{\partial^{2} \Psi_{T I}}{\partial \xi_{I} \otimes \partial \xi_{I}}\right)^{-1} \cdot\left(\frac{\partial^{2} U_{0 I}}{\partial \xi_{I} \otimes \partial \boldsymbol{G}}+\frac{\partial^{2} \Psi_{T I}}{\partial \xi_{I} \otimes \partial \boldsymbol{G}}\right)\right]\left.\right|_{\xi_{I}=\breve{\xi}_{I}}, \\
\boldsymbol{M}_{\boldsymbol{G F}}=\frac{\partial \boldsymbol{Q}_{T}}{\partial \boldsymbol{F}}= & \frac{1}{\Omega_{I}}\left[\left(\frac{\partial^{2} U_{0 I}}{\partial \boldsymbol{G} \otimes \partial \boldsymbol{F}}+\frac{\partial^{2} \Psi_{T I}}{\partial \boldsymbol{G} \otimes \partial \boldsymbol{F}}\right)-\left(\frac{\partial^{2} U_{0 I}}{\partial \boldsymbol{G} \otimes \partial \xi_{I}}+\frac{\partial^{2} \Psi_{T I}}{\partial \boldsymbol{G} \otimes \partial \xi_{I}}\right)\right. \\
& \left.\cdot\left(\frac{\partial^{2} U_{0 I}}{\partial \xi_{I} \otimes \partial \xi_{I}}+\frac{\partial^{2} \Psi_{T I}}{\partial \xi_{I} \otimes \partial \xi_{I}}\right)^{-1} \cdot\left(\frac{\partial^{2} U_{0 I}}{\partial \xi_{I} \otimes \partial \boldsymbol{F}}+\frac{\partial^{2} \Psi_{T I}}{\partial \xi_{I} \otimes \partial \boldsymbol{F}}\right)\right]\left.\right|_{\xi_{I}=\xi_{I}}
\end{aligned}
$$




$$
\begin{aligned}
\boldsymbol{M}_{\boldsymbol{G G}}=\frac{\partial \boldsymbol{Q}_{T}}{\partial \boldsymbol{G}}= & \frac{1}{\Omega_{I}}\left[\left(\frac{\partial^{2} U_{0 I}}{\partial \boldsymbol{G} \otimes \partial \boldsymbol{G}}+\frac{\partial^{2} \Psi_{T I}}{\partial \boldsymbol{G} \otimes \partial \boldsymbol{G}}\right)-\left(\frac{\partial^{2} U_{0 I}}{\partial \boldsymbol{G} \otimes \partial \xi_{I}}+\frac{\partial^{2} \Psi_{T I}}{\partial \boldsymbol{G} \otimes \partial \xi_{I}}\right)\right. \\
& \left.\cdot\left(\frac{\partial^{2} U_{0 I}}{\partial \xi_{I} \otimes \partial \xi_{I}}+\frac{\partial^{2} \Psi_{T I}}{\partial \xi_{I} \otimes \partial \xi_{I}}\right)^{-1} \cdot\left(\frac{\partial^{2} U_{0 I}}{\partial \xi_{I} \otimes \partial \boldsymbol{G}}+\frac{\partial^{2} \Psi_{T I}}{\partial \xi_{I} \otimes \partial \boldsymbol{G}}\right)\right]\left.\right|_{\xi_{I}=\check{\xi}_{I}}
\end{aligned}
$$

The interested reader can refer to Guo et al. $[21,30]$ for the explicit expressions of the terms in Eq. (18)-(24).

\section{Meshless numerical computational scheme}

In this section, based on the temperature-related multiscale QC constitutive model, the meshless numerical computational scheme is developed for the prediction of the buckling and post-buckling of SWCNCs at finite temperature.

\subsection{Moving least-squares interpolation}

For the construction of the numerical computational scheme, the moving least-squares approximation [31] is employed to describe the approximate displacement field which can naturally meet the higher-order continuity requirement of the THCB rule based constitutive model. Therefore, the approximated field can be calculated as

$$
\boldsymbol{u}=\sum_{I=1}^{N} \phi_{I}(\boldsymbol{X}) \widehat{\boldsymbol{u}}_{I},
$$

where $\widehat{\boldsymbol{u}}_{I}=\left(\hat{u}_{I 1}, \hat{u}_{I 2}, \hat{u}_{I 3}\right)^{T}$ is the nodal displacement parameter of Ith node. $\boldsymbol{\phi}(\boldsymbol{X})=$ $\left(\phi_{1}(\boldsymbol{X}), \phi_{2}(\boldsymbol{X}), \cdots, \phi_{N}(\boldsymbol{X})\right)$ is the meshless shape function, expressed as

$$
\phi_{I}(X)=\boldsymbol{p}(X) \cdot\left[A^{-1}(X) \cdot B(X)\right]_{I},
$$

where $\boldsymbol{p}(\boldsymbol{X})$ is the complete polynomial basis function, it takes the following form herein

$$
\boldsymbol{p}(\boldsymbol{X})=\left(1, X_{1}, X_{2}, X_{1}^{2}, X_{1} X_{2}, X_{2}\right)^{T},
$$


and

$$
\begin{gathered}
\boldsymbol{A}(\boldsymbol{X})=\sum_{I=1}^{n} \omega\left(\boldsymbol{X}-\boldsymbol{X}_{I}\right) \boldsymbol{p}\left(\boldsymbol{X}_{I}\right) \cdot \boldsymbol{p}\left(\boldsymbol{X}_{I}\right), \\
\boldsymbol{B}(\boldsymbol{X})=\left[\omega\left(\boldsymbol{X}-\boldsymbol{X}_{1}\right) \boldsymbol{p}\left(\boldsymbol{X}_{1}\right), \omega\left(\boldsymbol{X}-\boldsymbol{X}_{2}\right) \boldsymbol{p}\left(\boldsymbol{X}_{2}\right), \cdots, \omega\left(\boldsymbol{X}-\boldsymbol{X}_{n}\right) \boldsymbol{p}\left(\boldsymbol{X}_{n}\right)\right]
\end{gathered}
$$

where $\omega\left(\boldsymbol{X}-\boldsymbol{X}_{I}\right)$ is the weight function. $n$ is the number of nodes in the compactly support domain.

The first and second partial derivatives of $\phi_{I}(\boldsymbol{X})$ can be described as

$$
\begin{gathered}
\phi_{I, K}=p_{j, K}\left(\boldsymbol{A}^{-1} \cdot \boldsymbol{B}\right)_{j I}+p_{j}\left(\boldsymbol{A}_{, K}^{-1} \cdot \boldsymbol{B}+\boldsymbol{A}^{-1} \cdot \boldsymbol{B}_{, K}\right)_{j I^{\prime}} \\
\phi_{I, K L}=p_{j, K L}\left(\boldsymbol{A}^{-1} \cdot \boldsymbol{B}\right)_{j I}+p_{j, K}\left(\boldsymbol{A}_{, L}^{-1} \cdot \boldsymbol{B}+\boldsymbol{A}^{-1} \cdot \boldsymbol{B}_{, L}\right)_{j I}+p_{j, L}\left(\boldsymbol{A}_{, K}^{-1} \cdot \boldsymbol{B}+\boldsymbol{A}^{-1} \cdot \boldsymbol{B}_{, K}\right)_{j I} \\
+p_{j}\left(\boldsymbol{A}_{, K L}^{-1} \cdot \boldsymbol{B}+\boldsymbol{A}_{, K}^{-1} \cdot \boldsymbol{B}_{, L}+\boldsymbol{A}_{L}^{-1} \cdot \boldsymbol{B}_{, K}+\boldsymbol{A}^{-1} \cdot \boldsymbol{B}_{, K L}\right)_{j I}
\end{gathered}
$$

where

$$
\begin{gathered}
\boldsymbol{A}_{, K}^{-1}=-\boldsymbol{A}^{-1} \cdot \boldsymbol{A}_{, K} \cdot \boldsymbol{A}^{-1}, \\
\boldsymbol{A}_{, K}=\sum_{I=1}^{n} \omega_{, K}\left(\boldsymbol{X}-\boldsymbol{X}_{I}\right) \boldsymbol{p}\left(\boldsymbol{X}_{I}\right) \cdot \boldsymbol{p}\left(\boldsymbol{X}_{I}\right), \\
\boldsymbol{B}_{, K}=\left[\omega_{, K}\left(\boldsymbol{X}-\boldsymbol{X}_{1}\right) \boldsymbol{p}\left(\boldsymbol{X}_{1}\right), \omega_{, K}\left(\boldsymbol{X}-\boldsymbol{X}_{2}\right) \boldsymbol{p}\left(\boldsymbol{X}_{2}\right), \cdots, \omega_{, K}\left(\boldsymbol{X}-\boldsymbol{X}_{n}\right) \boldsymbol{p}\left(\boldsymbol{X}_{n}\right)\right] \\
\boldsymbol{A}_{, K L}^{-1}=\boldsymbol{A}^{-1} \cdot \boldsymbol{A}_{, L} \cdot \boldsymbol{A}^{-1} \cdot \boldsymbol{A}_{, K} \cdot \boldsymbol{A}^{-1}-\boldsymbol{A}^{-1} \cdot \boldsymbol{A}_{, K L} \cdot \boldsymbol{A}^{-1}+\boldsymbol{A}^{-1} \cdot \boldsymbol{A}_{, K} \cdot \boldsymbol{A}^{-1} \cdot \boldsymbol{A}_{, L} \cdot \boldsymbol{A}^{-1}, \\
\boldsymbol{B}_{, K L}=\left[\omega_{, K L}\left(\boldsymbol{X}-\boldsymbol{X}_{1}\right) \boldsymbol{p}\left(\boldsymbol{X}_{1}\right), \omega_{, K L}\left(\boldsymbol{X}-\boldsymbol{X}_{2}\right) \boldsymbol{p}\left(\boldsymbol{X}_{2}\right), \cdots, \omega_{, K L}\left(\boldsymbol{X}-\boldsymbol{X}_{n}\right) \boldsymbol{p}\left(\boldsymbol{X}_{n}\right)\right]
\end{gathered}
$$

\subsection{Numerical computational scheme}

Based on the constructed temperature-related multiscale QC model, the total energy of the considered SWCNC can be given as

$$
\begin{gathered}
\Pi_{\mathrm{tot}}=\Pi_{\mathrm{int}}+\Pi_{\mathrm{ext}}, \\
\Pi_{\mathrm{int}}=\int_{\Omega} W_{T}(\boldsymbol{F}, \boldsymbol{G}) \mathrm{dV},
\end{gathered}
$$




$$
\Pi_{\mathrm{ext}}=-\int_{\Omega} \boldsymbol{u} \cdot \boldsymbol{b}_{0} \mathrm{dV}-\int_{\Gamma_{\mathrm{t}}} \boldsymbol{u} \cdot \boldsymbol{t}_{0}^{P} \mathrm{~d} S-\int_{\Gamma_{\mathrm{t}}} \nabla_{N} \boldsymbol{u} \cdot \boldsymbol{t}_{0}^{Q} \mathrm{~d} \mathrm{~S},
$$

where $\boldsymbol{b}_{0}, \boldsymbol{t}_{0}^{P}$ and $\boldsymbol{t}_{0}^{Q}$ are the body force density, boundary traction and boundary couple, respectively. The normal gradient operator $\nabla_{N}(\circ)=\nabla_{X}(\circ) \cdot N$.

Assuming all the fields smooth enough, the variation of $\Pi_{\text {tot }}$ with respect to $\delta \boldsymbol{u}$ can be expressed as

$$
\begin{aligned}
& \delta \Pi_{\text {tot }}(\boldsymbol{u} ; \delta \boldsymbol{u}) \\
& =\delta \Pi_{\mathrm{int}}(\boldsymbol{u} ; \delta \boldsymbol{u})+\delta \Pi_{\mathrm{ext}}(\delta \boldsymbol{u}) \\
& =\int_{\Omega} \boldsymbol{P}_{T}: \nabla_{\boldsymbol{X}} \delta \boldsymbol{u} \mathrm{dV}+\int_{\Omega} \boldsymbol{Q}_{T} \vdots \nabla_{\boldsymbol{X}} \nabla_{\boldsymbol{X}} \delta \boldsymbol{u} \mathrm{dV}-\int_{\Omega} \delta \boldsymbol{u} \cdot \boldsymbol{b}_{0} \mathrm{dV}-\int_{\Gamma_{\mathrm{t}}} \delta \boldsymbol{u} \cdot \boldsymbol{t}_{0}^{P} \mathrm{dS}-\int_{\Gamma_{\mathrm{t}}} \nabla_{N} \delta \boldsymbol{u} \cdot \boldsymbol{t}_{0}^{Q} \mathrm{~d} S \\
& =0 .
\end{aligned}
$$

The strong form equilibrium equation can be derived as [32]

$$
\begin{gathered}
\left(\boldsymbol{Q}_{T} \cdot \nabla \cdot \nabla-\boldsymbol{P}_{T} \cdot \nabla\right)+\boldsymbol{b}_{0}=\mathbf{0} \text { in } \Omega, \\
\left(\boldsymbol{P}_{T}-\left(\boldsymbol{Q}_{T} \cdot \nabla\right)\right) \cdot \boldsymbol{N}+\boldsymbol{N} \nabla: \overline{\boldsymbol{T}}\left(\boldsymbol{Q}_{T} \cdot \boldsymbol{N}\right) \cdot \boldsymbol{N}-\left(\boldsymbol{Q}_{T} \cdot \boldsymbol{N}\right) \nabla: \overline{\boldsymbol{T}}=\boldsymbol{t}_{0}^{P} \quad \text { on } \Gamma_{\mathrm{t}}, \\
\boldsymbol{Q}_{T}:(\boldsymbol{N} \otimes \boldsymbol{N})=\boldsymbol{t}_{0}^{Q} \quad \text { on } \Gamma_{\mathrm{t}},
\end{gathered}
$$

where $\bar{T}=\boldsymbol{I}-\boldsymbol{N} \otimes \boldsymbol{N}$.

From Eq. (40)-(43), it is clear that the virtual work representation of equilibrium equation is a nonlinear function of $\boldsymbol{u}$. To obtained the equilibrium solution, Newton-Raphson iteration [33] can be used to solve the problem in an increment way. The incremental equation of system can be derived from a linearization process of Eq. (40) as

$$
\delta \Pi_{\text {tot }}(\boldsymbol{u} ; \delta \boldsymbol{u}) \cong \delta \Pi_{\text {tot }}\left(\boldsymbol{u}^{i} ; \delta \boldsymbol{u}\right)+\mathrm{D} \delta \Pi_{\text {tot }}\left(\boldsymbol{u}^{i} ; \delta \boldsymbol{u}\right)[\Delta \boldsymbol{u}]=0 \text {, }
$$

where $\Delta \boldsymbol{u}$ is the incremental displacements after $i-$ th iteration.

Taking into account the fact that 


$$
\operatorname{D} \delta \Pi_{e x t}(\delta \boldsymbol{u})[\Delta \boldsymbol{u}]=0
$$

$$
\begin{aligned}
& \mathrm{D} \delta \Pi_{\text {tot }}\left(\boldsymbol{u}^{i} ; \delta \boldsymbol{u}\right)[\Delta \boldsymbol{u}]=\mathrm{D} \delta \Pi_{\text {int }}\left(\boldsymbol{u}^{i} ; \delta \boldsymbol{u}\right)[\Delta \boldsymbol{u}] \\
& =\int_{\Omega} \mathrm{D}\left(\boldsymbol{P}_{T}: \nabla_{\boldsymbol{X}} \delta \boldsymbol{u}\right)[\Delta \boldsymbol{u}] \mathrm{dV}+\int_{\Omega} \mathrm{D}\left(\boldsymbol{Q}_{T} \vdots \nabla_{\boldsymbol{X}} \nabla_{\boldsymbol{X}} \delta \boldsymbol{u}\right)[\Delta \boldsymbol{u}] \mathrm{dV} \\
& =\int_{\Omega} \nabla_{\boldsymbol{X}} \delta \boldsymbol{u}: \mathrm{D} \boldsymbol{P}_{T}[\Delta \boldsymbol{u}]+\boldsymbol{P}_{T}: \mathrm{D}\left(\nabla_{\boldsymbol{X}} \delta \boldsymbol{u}\right)[\Delta \boldsymbol{u}]+\nabla_{\boldsymbol{X}} \nabla_{\boldsymbol{X}} \delta \boldsymbol{u} \vdots \mathrm{D}_{T}[\Delta \boldsymbol{u}]+\boldsymbol{Q}_{T} \vdots \mathrm{D}\left(\nabla_{\boldsymbol{X}} \nabla_{\boldsymbol{X}} \delta \boldsymbol{u}\right)[\Delta \boldsymbol{u}] \mathrm{dV} \\
& =\int_{\Omega} \nabla_{\boldsymbol{X}} \delta \boldsymbol{u}: \mathrm{D}_{T}[\Delta \boldsymbol{u}]+\nabla_{\boldsymbol{X}} \nabla_{\boldsymbol{X}} \delta \boldsymbol{u} \vdots \mathrm{D} \boldsymbol{Q}_{T}[\Delta \boldsymbol{u}] \mathrm{dV}
\end{aligned}
$$

From Eq. (21)-(24), DP $\boldsymbol{P}_{T}[\Delta \boldsymbol{u}]$ and $\mathrm{D} \boldsymbol{Q}_{T}[\Delta \boldsymbol{u}]$ in Eq. (46) can be further written as

$$
\begin{aligned}
& \mathrm{D}_{T}[\Delta \boldsymbol{u}]=\frac{\mathrm{D} \boldsymbol{P}_{T}}{\mathrm{D} \boldsymbol{F}}: \mathrm{D} \boldsymbol{F}[\Delta \boldsymbol{u}]+\frac{\mathrm{D} \boldsymbol{P}_{T}}{\mathrm{D} \boldsymbol{G}}: \mathrm{D} \boldsymbol{G}[\Delta \boldsymbol{u}]=\boldsymbol{M}_{\boldsymbol{F} F}: \nabla_{\boldsymbol{X}} \Delta \boldsymbol{u}+\boldsymbol{M}_{\boldsymbol{F} \boldsymbol{G}} \vdots \nabla_{\boldsymbol{X}} \nabla_{\boldsymbol{X}} \Delta \boldsymbol{u}, \\
& \mathrm{D} \boldsymbol{Q}_{T}[\Delta \boldsymbol{u}]=\frac{\mathrm{D} \boldsymbol{Q}_{T}}{\mathrm{D} \boldsymbol{F}}: \mathrm{D} \boldsymbol{F}[\Delta \boldsymbol{u}]+\frac{\mathrm{D} \boldsymbol{Q}_{T}}{\mathrm{D} \boldsymbol{G}} \vdots \mathrm{D} \boldsymbol{G}[\Delta \boldsymbol{u}]=\boldsymbol{M}_{\boldsymbol{G} \boldsymbol{F}}: \nabla_{\boldsymbol{X}} \Delta \boldsymbol{u}+\boldsymbol{M}_{\boldsymbol{G} \boldsymbol{G}} \vdots \nabla_{\boldsymbol{X}} \nabla_{\boldsymbol{X}} \Delta \boldsymbol{u} .
\end{aligned}
$$

Inserting Eq. (47) and (48) into Eq. (46) yields

$$
\begin{aligned}
& \mathrm{D} \delta \Pi_{\text {tot }}\left(\boldsymbol{u}^{i} ; \delta \boldsymbol{u}\right)[\Delta \boldsymbol{u}] \\
& =\int_{\Omega} \nabla_{\boldsymbol{X}} \delta \boldsymbol{u}: \boldsymbol{M}_{\boldsymbol{F F}}: \nabla_{\boldsymbol{X}} \Delta \boldsymbol{u}+\nabla_{\boldsymbol{X}} \delta \boldsymbol{u}: \boldsymbol{M}_{\boldsymbol{F} G}: \nabla_{\boldsymbol{X}} \nabla_{\boldsymbol{X}} \Delta \boldsymbol{u}+\nabla_{\boldsymbol{X}} \nabla_{\boldsymbol{X}} \delta \boldsymbol{u}: \boldsymbol{M}_{G F}: \nabla_{\boldsymbol{X}} \Delta \boldsymbol{u}+\nabla_{\boldsymbol{X}} \nabla_{\boldsymbol{X}} \delta \boldsymbol{u} \boldsymbol{M}_{G G} \\
& \vdots \nabla_{\boldsymbol{X}} \nabla_{\boldsymbol{X}} \Delta \boldsymbol{u} \mathrm{dV} \\
& =\delta \widehat{\boldsymbol{u}} \cdot\left[\int_{\Omega} \nabla_{X} \boldsymbol{\phi} \cdot \boldsymbol{M}_{F F} \cdot \nabla_{X} \boldsymbol{\phi}+\nabla_{X} \boldsymbol{\phi} \cdot \boldsymbol{M}_{F G}: \nabla_{X} \nabla_{X} \boldsymbol{\phi}+\nabla_{X} \nabla_{X} \boldsymbol{\phi}: \boldsymbol{M}_{G F} \cdot \nabla_{X} \boldsymbol{\phi}\right. \\
& \left.+\nabla_{X} \nabla_{X} \boldsymbol{\phi}: \boldsymbol{M}_{G G}: \nabla_{X} \nabla_{X} \boldsymbol{\phi d V}\right] \cdot \Delta \widehat{\boldsymbol{u}}
\end{aligned}
$$$$
=\delta \widehat{\boldsymbol{u}} \cdot \boldsymbol{K}\left(\boldsymbol{u}^{i}\right) \cdot \Delta \widehat{\boldsymbol{u}}
$$

From Eq. (25), $\delta \Pi_{\text {tot }}\left(\boldsymbol{u}^{i} ; \delta \boldsymbol{u}\right)$ can be further written as

$$
\begin{aligned}
& \delta \Pi_{\text {tot }}\left(\boldsymbol{u}^{i} ; \delta \boldsymbol{u}\right) \\
& =\int_{\Omega} \delta \widehat{\boldsymbol{u}} \cdot \boldsymbol{P}_{T} \cdot \nabla_{\boldsymbol{X}} \boldsymbol{\phi}+\delta \widehat{\boldsymbol{u}} \cdot \boldsymbol{Q}_{T}: \nabla_{\boldsymbol{X}} \nabla_{\boldsymbol{X}} \boldsymbol{\phi}-(\delta \widehat{\boldsymbol{u}} \cdot \boldsymbol{\phi}) \cdot \boldsymbol{b}_{0} \mathrm{dV}-\int_{\Gamma_{\mathrm{t}}}(\delta \widehat{\boldsymbol{u}} \cdot \boldsymbol{\phi}) \cdot \boldsymbol{t}_{0}^{P} \mathrm{~d} \mathrm{~S} \\
& -\int_{\Gamma_{\mathrm{t}}} \delta \widehat{\boldsymbol{u}} \cdot\left(\nabla_{X} \boldsymbol{\phi} \cdot \boldsymbol{N}\right) \boldsymbol{t}_{0}^{Q} \mathrm{dS} \\
& =\delta \widehat{\boldsymbol{u}} \cdot\left[\int_{\Omega} \boldsymbol{P}_{T} \cdot \nabla_{\boldsymbol{X}} \boldsymbol{\phi}+\boldsymbol{Q}_{T}: \nabla_{\boldsymbol{X}} \nabla_{\boldsymbol{X}} \boldsymbol{\phi}-\boldsymbol{\phi} \boldsymbol{b}_{0} \mathrm{dV}-\int_{\Gamma_{\mathrm{t}}} \boldsymbol{\phi} \boldsymbol{t}_{0}^{P} \mathrm{dS}-\int_{\Gamma_{\mathrm{t}}}\left(\nabla_{\boldsymbol{X}} \boldsymbol{\phi} \cdot \boldsymbol{N}\right) \boldsymbol{t}_{0}^{Q} \mathrm{dS}\right]
\end{aligned}
$$


$=-\delta \widehat{\boldsymbol{u}} \cdot \boldsymbol{f}$.

Substitution of Eq. (49) and (50) into Eq. (44) yields

$$
\delta \widehat{\boldsymbol{u}} \cdot\left(\boldsymbol{K}\left(\boldsymbol{u}^{i}\right) \cdot \Delta \widehat{\boldsymbol{u}}-\boldsymbol{f}\right)=0,
$$

for $\forall \delta \widehat{\boldsymbol{u}}$,

$$
\boldsymbol{K}\left(\boldsymbol{u}^{i}\right) \cdot \Delta \widehat{\boldsymbol{u}}=\boldsymbol{f} .
$$

In this study, the Newton-Raphon method is used to find the equilibrium configurations of SWCNCs at finite temperature iteratively. It should be noted that the Newton-Raphson method fails when the global stiffness matrix $\boldsymbol{K}$ becomes non-positive definite at the point of buckling occurrence. Generally, the $\alpha$-method $\boldsymbol{K}+\alpha \boldsymbol{I}$ [34] is employed to overcome this tiresome problem. However, due to the involvement of the temperature effect herein, the issue becomes more complicated. Under this circumstance, the modified $\alpha$-method [22] in which $\boldsymbol{K}$ (temperature effect considered) is replaced with $\boldsymbol{K}^{0}$ (temperature effect not considered) is resorted to settle this trouble. The effectiveness of the modified $\alpha$-method for solving buckling and post-buckling problems in thermal environments has been confirmed by Wang and Guo [22-24].

\section{Numerical examples and discussion}

\subsection{Rationality and validity of the proposed numerical scheme}

In this subsection, several numerical examples provided to investigate the rationality and validity of proposed numerical scheme for the prediction of deformation behaviors of SWCNCs under compression at finite temperature. In all the numerical examples, the open-tip SWCNCs are selected and the loading process is treated as isothermal and 
quasi-static. Before holding and loading, an energy minimization process with respect to the relaxation parameters $\xi_{I}(T)$ and $\lambda(T)=\left(\lambda_{1}(T), \lambda_{2}(T)\right)^{T}$ should be adopted to insure the initial equilibrium of the SWCNC which is rolling up from the fan-shaped graphene sheet counterpart. Subsequently, the bottom end of the cone is fixed and the axial compression is imposed on the top end (the free end) incrementally. Simultaneously, the in-plane displacements of the top end are prohibited. The penalty method is utilized to enforce the essential boundary conditions.

The geometry of the open-tip SWCNC is shown in Fig. 5. $\varphi$ is the apex angle of the SWCNC, $r_{c}$ is the radius of the top end and $H_{c}$ is the height of the open-tip SWCNC. The meshless nodes are distributed on the surface of SWCNC as uniformly as possible. $(m \times n)$ is the control parameter for the distribution of meshless nodes in which $m$ nodes are located evenly along the side edge and $n$ nodes are placed uniformly on the top circle of the cone. Due to the radius of SWCNCs enlarging along the height direction, the number of circumferential nodes increases incrementally from $n$. The rotation angle of cutting lines of graphene sheet is set as $0^{\circ}$ (see Fig. 1 for reference), unless otherwise stated.

Firstly, the open-tip SWCNC with the apex angle $\varphi=19.2^{\circ}$, the height $H_{c}=5.92 \mathrm{~nm}$ and the top radius $r_{c}=0.67 \mathrm{~nm}$ is taken to study the influence of the number of meshless nodes on the numerical simulation at 300K. Fig. 6 shows the comparison the strain energy per atom of the SWCNC with different number of meshless nodes used from 490 $(20 \times 15), 708(24 \times 18)$ and $938(28 \times 20)$ (values in parentheses denote the node control parameters). It can be noticed that the strain energy per atom climbs in an 
approximate quadratic form with the axial compression strain increasing before the first buckling occurs which is corresponding to the first strain energy abrupt drop, after which the strain energy increases linearly up to the second abrupt drop (i.e. the post-buckling stage). The first critical strains are 3.37\%, 3.30\% and 3.27\%, respectively, with the node number enlarging from 490 to 938 . The corresponding values of the strain energy drops are $0.0039 \mathrm{eV}, 0.0036 \mathrm{eV}$ and $0.0036 \mathrm{eV}$, respectively. The second critical strains corresponding to the post-buckling are 3.79\%, 3.76\% and $3.75 \%$ and the values of the energy drops are $0.00101 \mathrm{eV}, 0.001 \mathrm{eV}$ and $0.001 \mathrm{eV}$, respectively. By comparison, with the number of meshless nodes increasing, the first and second critical strains are gradually close to about $3.2 \%$ and $3.7 \%$ and the values of the energy drops are close to $0.0036 \mathrm{eV}$ and $0.001 \mathrm{eV}$, respectively, which is reasonable from the numerical point of view. The values of the energy drops reduce from $0.0036 \mathrm{eV}$ to $0.001 \mathrm{eV}$ with the increase of the axial compression strain, and this tendency is in a good agreement with Liew et al. [4].

For further checking the validity of the present numerical scheme, the example studied by Yan et al. [8] is selected in which the apex angle $\varphi=19.2^{\circ}$, the height $H_{c}=7.37 \mathrm{~nm}$ and the top radius $r_{c}=0.6 \mathrm{~nm}$. Since the temperature effect is not considered in the literature [8], for ease of comparison, the system temperature is set as 1K herein. $663(26 \times 13)$ meshless nodes are used for the numerical simulation. Fig. 7 compares the strain energy per atom provided by the present method and Yan et al. [8] for the considered open-tip SWCNC under axial compression. As can be seen, the numerical result obtained from the present method agrees well with those reported in Ref. [8]. The buckling occurs at the axial compressive ratio of $2.98 \%$, and the corresponding buckling 
pattern is depicted in Fig. 8 which is very comparable with that presented in Ref. [4] (MD simulation) and [8] (QC model). It should be pointed out that compared with the MD simulation which needs 2106 atoms, the present approach with use of only 663 meshless nodes can provide very reliable results.

Furthermore, the influence of temperature on the mechanical responses of open-tip SWCNCs is also inspected in the present subsection. The numerical example investigated by Liao [35] is selected as the reference, and the geometrical parameters the open-tip SWCNC are $\varphi=19.2^{\circ}, H_{c}=3.04 \mathrm{~nm}$ and $r_{c}=0.34 \mathrm{~nm}$, respectively. The three prescribed system temperatures $300 \mathrm{~K}, 500 \mathrm{~K}$ and $700 \mathrm{~K}$ are used for the numerical simulation. Fig. 9 shows the variation of the strain energy per atom with respect to axial compression strain at temperatures of $300 \mathrm{~K}, 500 \mathrm{~K}$ and $700 \mathrm{~K}$, respectively. It can be found that the temperature has a decreasing effect on the buckling resistance of SWCNC. The critical strains are $5.0 \%, 4.9 \%$ and $4.8 \%$ at $300 \mathrm{~K}, 500 \mathrm{~K}$ and $700 \mathrm{~K}$, respectively, which are very close to $5.2 \%, 5.1 \%$ and $5.0 \%$, respectively, calculated though MD simulation in Ref. [35].

Consequently, the proposed temperature-related multiscale QC model and the corresponding numerical scheme are effective and efficient for analyzing the buckling of open-tip SWCNCs at finite temperature. With use of the present method, the axial compressive buckling behaviors of open-tip SWCNCs are discussed systematically in the following section. The system temperature is set as $300 \mathrm{~K}$, unless otherwise stated.

\subsection{The effect of rotation angle $\theta_{r}$ on buckling of SWCNCS}


In this subsection, the open-tip SWCNCs formed by different fan-shaped graphene sheets in which the rotation angles of the cutting lines vary from $-30^{\circ}$ to $30^{\circ}$ (see section 2.2 and Fig. 1 for reference) are selected to investigate the effect of rotation angle on the buckling of SWCNCs. The geometrical parameters of the open-tip SWCNCs are selected as $\varphi=19.2^{\circ}, H_{c}=6.9 \mathrm{~nm}$, and $r_{c}=0.33 \mathrm{~nm}$. The number of meshless nodes are taken as $816(32 \times 10)$. The variation of critical compression strain with respect to rotation angle is depicted in Fig. 10. Clearly, numerical results obtained indicate that the critical load of buckling decreases as the value of $\theta_{r}$ varies from $0^{\circ}$ to $30^{\circ}$. The same conclusion also holds when $\theta_{r}$ varies from $0^{\circ}$ to $-30^{\circ}$ (i.e., the SWCNC with $\theta_{r}=0^{\circ}$ has the strongest buckling resistance ability). The critical strains are 4.08\%, 4.05\%, 3.98\%, $3.84 \%, 3.69 \%, 3.62 \%$ and $3.55 \%$ with respect to $\theta_{r}$ from $0^{\circ}$ to $30^{\circ}$ and $4.08 \%, 3.94 \%$, $3.8 \%, 3.65 \%, 3.55 \% 3.45 \%$ and $3.43 \%$ from $0^{\circ}$ to $-30^{\circ}$, respectively. The largest difference of the critical strains is $0.19 \%$ between $3.84 \%$ and $3.65 \%$ with respect to $\theta_{r}$ of $-15^{\circ}$ and $15^{\circ}$, respectively. Although the micro-structures of open-tip SWCNCs/fan-shaped graphene sheets are symmetrical about $0^{\circ}$ rotation angle, the values of critical compression strain are not fully symmetrical, which might be ascribed to the error of numerical computations.

\subsection{The effect of height and radius on buckling of SWCNCS}

For the investigation of the height and radius effect on the buckling of open-tip SWCNCs, three groups of SWCNCs with different top radii of $0.5 \mathrm{~nm}, 0.83 \mathrm{~nm}$ and $1.167 \mathrm{~nm}$, respectively, are selected as samples in the simulation. The heights of the SWCNCs vary in 
a large range from $3.9 \mathrm{~nm}$ to $12.8 \mathrm{~nm}$. The numerical results are depicted in Fig. 11 . It demonstrates that although the critical strains grow slightly in the small range of $8 \mathrm{~nm}<H_{c}<11 \mathrm{~nm}$, they present downtrend basically with the increase of the height. In other words, SWCNCs become much easier to buckling as the height enlarges. It also can be seen that with the top radius increasing, the influence of the height on the critical compression strain becomes less (i.e. the critical strain curves become relatively flatter). It is attributed to the fact that as the top radius enlarges, the mechanical responses of SWCNCs are close to those of graphene, which is also confirmed in [8]. From another point of view, for the same height, the top radius has a decreasing effect on the anti-buckling ability of SWCNCs (i.e. the critical compression strain decreases with the increase of the top radius). It is also noticed that the influence of the top radius on the critical compression strain becomes weaken for the case of SWCNCs with large height.

To be clear, the effect of top radius on the buckling of open-tip SWCNCs is further studied and the variations of the critical compression strain with respect to the top radius for the open-tip SWCNCs with a same height of $5.92 \mathrm{~nm}$ are presented in Fig. 12. As can be observed from the figure, the critical compression strains decrease approximate quadratically and the curve gradually becomes gentle as the top radius enlarges from $0.5 \mathrm{~nm}$ to $1.5 \mathrm{~nm}$. Predictably, with the top radius continue increasing, the critical strain will be close to that of graphene.

In a word, both the top radius and the height have decreasing effect on the buckling resistance ability of open-tip SWCNCs. 
In this subsection, the influence of apex angle on the buckling of open-tip SWCNCs is investigated with use of the proposed numerical scheme. For comparison purpose, the open-tip SWCNCs with the same top radius $r_{c}=1.0 \mathrm{~nm}$ and side edge length (i.e. the length of the conical bus bar) taken as $6 \mathrm{~nm}$. Fig. 13 depicts the variation of the critical compression strain with respect to the apex angle for the considered SWCNCs. The critical compression strains are $2.53 \%, 1.77 \%, 1.92 \%, 2.21 \%$ and $2.74 \%$ corresponding to the apex angles of $19.2^{\circ}, 38.9^{\circ}, 60^{\circ}, 84.6^{\circ}$ and $112.9^{\circ}$, respectively. Except the SWCNC with $\varphi=19.2^{\circ}$, the critical compression strain increases with the apex angle enlarging, namely, the apex angle has an increasing effect on the buckling resistance ability. The corresponding buckling patterns (both front views and top views) are shown in Fig. 14 . An interesting finding is that all the buckling configurations are three fins modes and there are obvious differences between them. The buckling modes for SWCNCs with smaller apex angles are relatively regular, however, for the case of larger apex angles, the distribution of the pits on the surface of SWCNCs becomes random and asymmetrically which is like the ripples of graphene [36].

\section{Concluding remarks}

In the present study, a meshless numerical framework for the prediction of buckling and post-buckling of SWCNCs at finite temperature is developed based on the proposed temperature-related multiscale QC model. The distinguishing feature of the present QC model is the employment of the THCB rule as the linkage between the microscopic and macroscopic deformation, meanwhile, the curvature effect of SWCNCs can be taken into 
consideration in a convenient way. The degree of freedom of SWCNCs can be chosen freely and the computational efforts can be saved greatly compared with atomistic-based simulations. From the numerical results, the buckling and post-buckling of SWCNCs at finite temperature can be captured precisely. The rotation angle of cutting lines, the height and the top radius all have decreasing effect on the buckling resistance ability of SWCNCs. Furthermore, with the increase of apex angle, the buckling patterns of SWCNCs become irregular and are close to those of graphene. 


\section{Acknowledgements}

The financial support from the Promotive Research Fund for Excellent Young and Middle-aged Scientists of Shandong Province, China (BS2014SF004), the Natural Science Foundation of Shandong Province, China (ZR2015PE010), the Doctoral Starting up Foundation of Ludong University, China (LY2014022), National Natural Science Foundation (91216201 and 11402048), China Postdoctoral Science Foundation (2014M561221), Program for Changjiang Scholars, Innovative Research Team in University (PCSIRT) and 111 Project (B14013), the National Science Foundation of China (11372281), the province Science Foundation of Zhejiang (LY13A020003) are gratefully acknowledged. 


\section{References}

[1] R. Ansari, A. Momen, S. Rouhi, S. Ajori, On the vibration of single-walled carbon nanocones: molecular mechanics approach versus molecular dynamics simulations, Shock Vib. 2014 (2014) 410783.

[2] M.M.S. Fakhrabadi, N. Khani, R. Omidvar, A. Rastgoo, Investigation of elastic and buckling properties of carbon nanocones using molecular mechanics approach, Comput. Mater. Sci. 61 (2012) 248-256.

[3] S.P. Jordan, V.H. Crespi, Theory of carbon nanocones: mechanical chiral inversion of a micron-scale three-dimensional object, Phys. Rev. Lett. 93 (2004) 255504.

[4] K.M. Liew, J.X. Wei, X.Q. He, Carbon nanocones under compression: Buckling and post-buckling behaviors, Phys. Rev. B 75 (2007) 195435.

[5] J.H. Lee, B.S. Lee, Modal analysis of carbon nanotubes and nanocones using FEM, Comput. Mater. Sci. 51 (2012) 30-42.

[6] C. Wei, D. Srivastava, Nanomechanics of carbon nanofibers: Structural and elastic properties, Appl. Phys. Lett. 85 (2004) 2208-2210.

[7] J.W. Yan, K.M. Liew, L.H. He, Predicting mechanical properties of single-walled carbon nanocones using a higher-order gradient continuum computational framework, Compos. Struct. 94 (2012) 3271-3277.

[8] J.W. Yan, K.M. Liew, L.H. He, A mesh-free computational framework for predicting buckling behaviors of single-walled carbon nanocones under axial compression based on the moving Kriging interpolation, Comput. Methods Appl. Mech. Engrg. 247-248 (2012) 103-112. 
[9] J.W. Yan, K.M. Liew, L.H. He, Buckling and post-buckling of single-wall carbon nanocones upon bending, Compos. Struct. 106 (2013) 793-798.

[10] J.W. Yan, K.M. Liew, Predicting elastic properties of single-walled boron nitride nanotubes and nanocones using an atomistic-continuum approach. Compos. Struct. 125 (2015) 489-498.

[11] R. Ansari, E. Mahmoudinezhad, Characterizing the mechanical properties of carbon nanocones using an accurate spring-mass model. Computational Materials Science 101 (2015) 260-266.

[12] R. Ansari, H. Rouhi, A. Nasiri Rad, Vibrational analysis of carbon nanocones under different boundary conditions: An analytical approach. Mechanics Research Communications, 56 (2014) 130-135.

[13] R. Ansari, F. Alisafaei, A. Alipour, E. Mahmoudinezhad, On the van der Waals interaction of carbon nanocones. Journal of Physics and Chemistry of Solids 73 (2012) 751-756.

[14] R. Ansari, M. Hosseinzadeh, Mechanics of single-walled carbon nanotubes inside open single-walled carbon nanocones, Journal of Mechanical Science and Technology 27 (2013) 3363-3370.

[15] R. Ansari, F. Sadeghi, On the mechanics of C60 fullerene inside open carbon nanocones: A continuum study. Physica E: Low-dimensional Systems and Nanostructures. $69(2015) 1-12$.

[16] R.D. Firouz-Abadi, M.M. Fotouhi, H. Haddadpour, Stability analysis of nanocones under external pressure and axial compression using a nonlocal shell model. Physica E: 
Low-dimensional Systems and Nanostructures 44 (2012) 1832-1837.

[17] K.B. Mustapha, Z.W. Zhong, Stability of single-walled carbon nanotubes and single-walled carbon nanocones under self-weight and an axial tip force. International Journal of Engineering Science 50 (2012) 268-278.

[18] P.C. Tsai, T.F. Fang, A molecular dynamics study of the nucleation, thermal stability and nanomechanics of carbon nanocones, Nanotech. 18 (2007) 105702.

[19] M.L. Li, F. Lin, Y. Chen, Study on the mechanical properties of carbon nanocones using molecular dynamics simulation, Acta Phys. Sin. 62 (2013) 016102.

[20] M.L. Liao, C.H. Cheng, Y.P. Lin, Tensile and compressive behaviors of open-tip carbon nanocones under axial strains, J. Mater. Res. 26 (2011) 1577-1584.

[21] X. Guo, J.B. Liao, X.Y. Wang, Investigation of the thermo-mechanical properties of single-walled carbon nanotubes based on the temperature-related higher order Cauchy-Born rule, Comput. Mater. Sci. 51 (2012) 445-454.

[22] X.Y. Wang, X. Guo, Numerical simulation for finite deformation of single-walled carbon nanotubes at finite temperature using temperature-related higher order Cauchy-Born rule based quasi-continuum model, Comput. Mater. Sci. 55 (2012) 273-283. [23] X.Y. Wang, X. Guo, Quasi-continuum model for the finite deformation of single-layer graphene sheets based on the temperature-related higher order Cauchy-Born rule, $\boldsymbol{J}$. Comput. Theor. Nanosci. 10 (2013) 154-164.

[24] X.Y. Wang, X. Guo, Quasi-continuum contact model for the simulation of severe deformation of single-walled carbon nanotubes at finite temperature, J. Comput. Theor. Nanosci. 10 (2013) 810-820. 
[25] J. Dolbow, T. Belytschko, An introduction to programming the meshless element free Galerkin method, Arch. Comput. Methods Eng. 5 (1998) 207-241.

[26] A. Krishnan, E. Dujardin, M.M.J. Treacy, J. Hugdahl, S. Lynum, T.W. Ebbesen, Graphitic cones and the nucleation of curved carbon surfaces, Nature 388 (1997) 451-454.

[27] H. Jiang, Y. Huang, K.C. Hwang, A finite-temperature continuum theory based on interatomic potentials, J. Eng. Mater. Technol. 127 (2005) 408-416.

[28] J. Tersoff, New empirical approach for the structure and energy of covalent systems, Phys. Rev. B 37 (1988) 6991-7000.

[29] D.W. Brenner, Empirical potential for hydrocarbons for use in simulating the chemical vapor deposition of diamond films, Phys. Rev. B 42 (1990) 9458-9471.

[30] X. Guo, J.B. Wang, H.W. Zhang, Mechanical properties of single-walled carbon nanotubes based on higher order Cauchy-Born rule, Int. J. Solids Struct. 43 (2006) 1276-1290.

[31] P. Lancaster, K. Salkauskas, Surfaces generated by moving least squares methods, Math. Comput. 37 (1981) 141-158.

[32] R. Sunyk, P. Steinmann, On higher gradients in continuum-atomistic modeling, Int. J. Solids Struct. 40 (2003) 6877-6896.

[33] J.E. Dennis, R.B. Schnabel, Numerical Methods for Unconstrained Optimization and Nonlinear Equations, Prentice-Hall, Englewood Cliffs, NJ, 1983.

[34] B. Liu, Y. Huang, H. Jiang, S. Qu, K.C. Hwang, The atomic-scale finite element method, Comput. Methods Appl. Mech. Eng. 193 (2004) 1849-1864.

[35] M.L. Liao, Buckling behaviors of open-tip carbon nanocones at elevated temperatures, 
Appl. Phys. A 117 (2014) 1109-1118.

[36] A. Fasolino, J.H. Los, M.I. Katsnelson, Intrinsic ripples in graphene, Nat. Mater. 6 (2007) 858-861. 


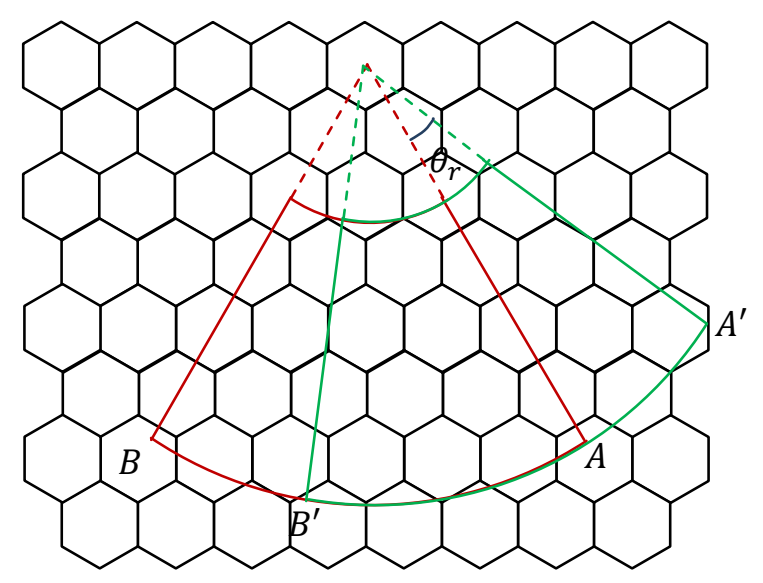

Fig. 1. Schematic illustration of clipping of fan-shaped graphene sheet. 

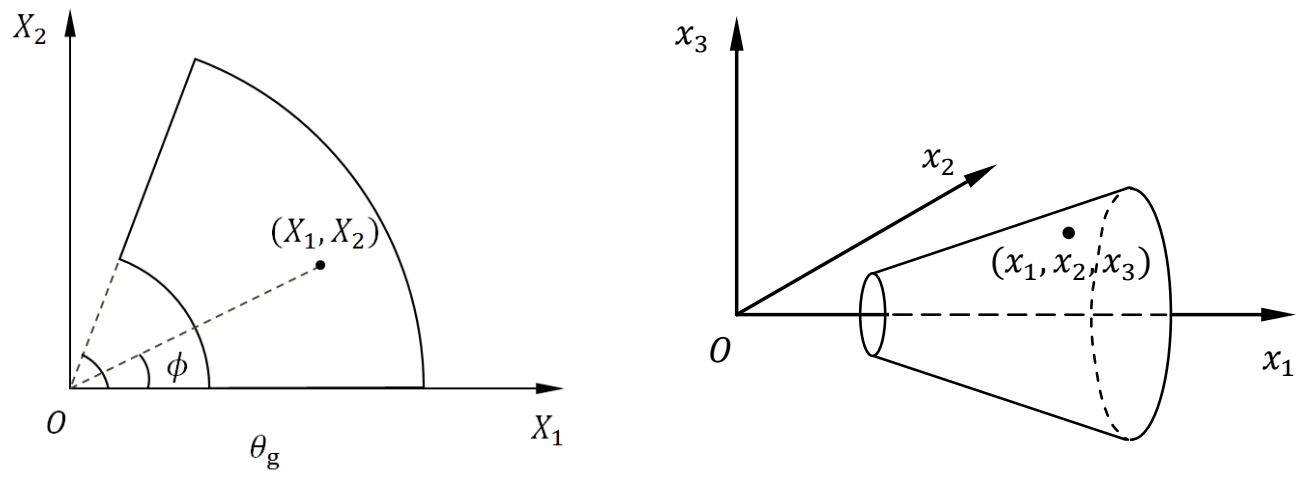

Fig. 2. Transformation mapping from planar fan-shaped graphene sheet to the undeformed SWCNC: (a) a planar fan-shaped graphene sheet, (b) the undeformed SWCNC rolled from the planar fan-shaped graphene sheet. 


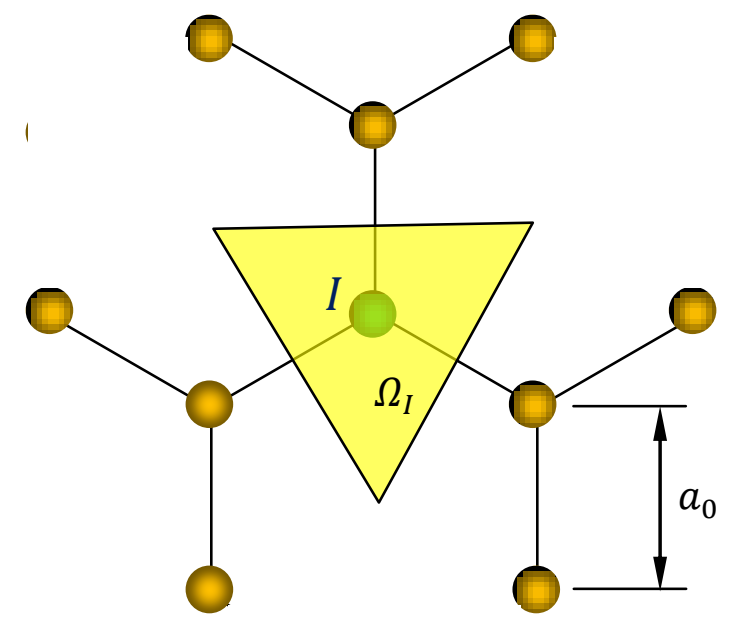

Fig. 3. The representative cell associated with atom $I$. 


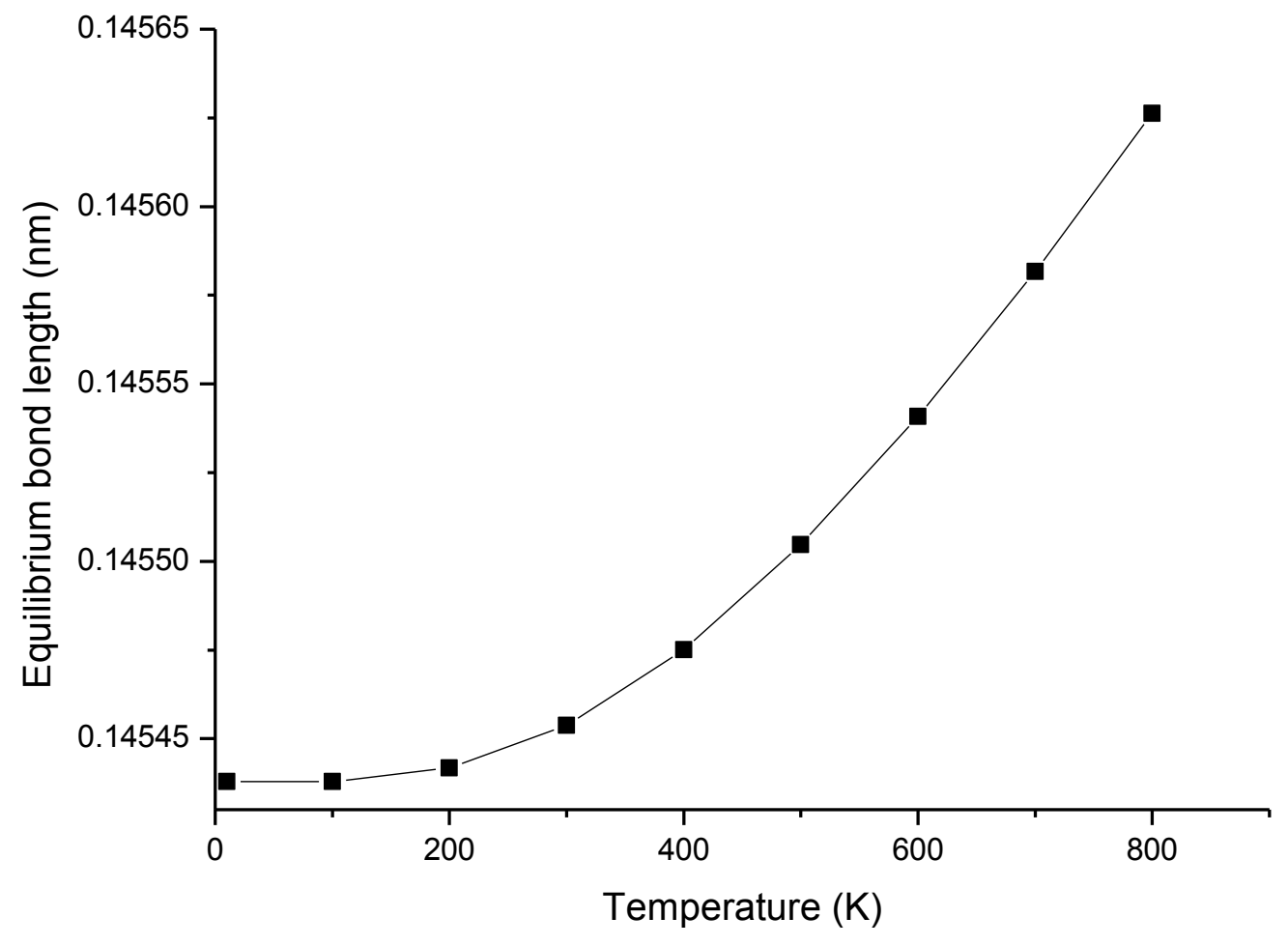

Fig. 4. Variation of the equilibrium bond length with respect to temperature. 


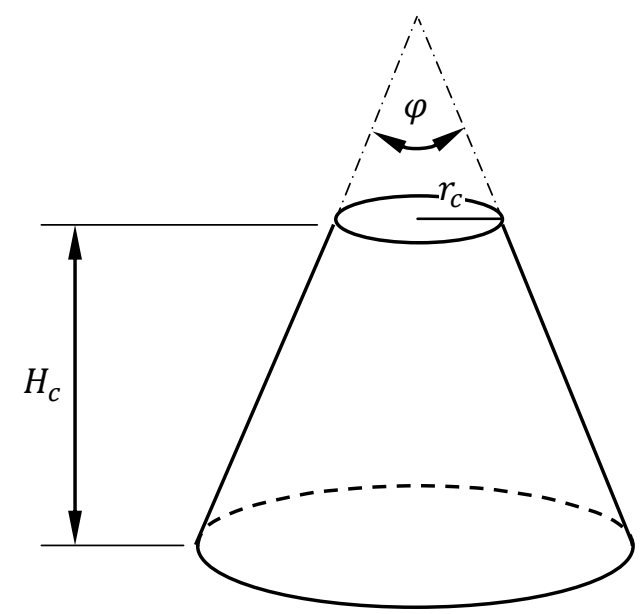

Fig. 5. Schematic illustration of the open-tip SWCNC: $\varphi$ is the apex angle of the SWCNC, $r_{C}$ is the radius of the top end and $H_{C}$ is the height of the open-tip SWCNC, respectively. 


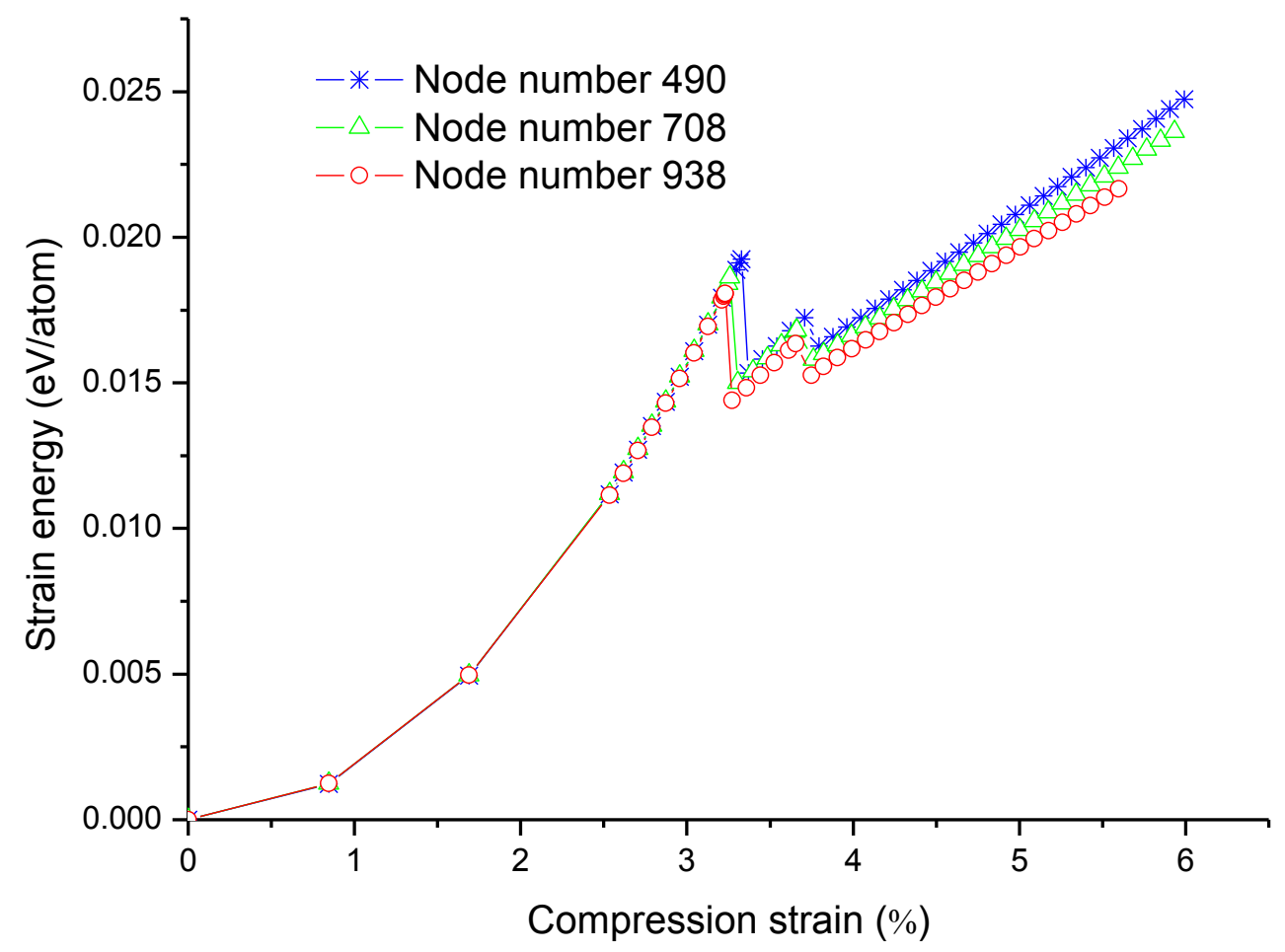

Fig. 6. The strain energy versus the axial compression strain with the use of 490 $(20 \times 15), 708(24 \times 18)$ and $938(28 \times 20)$ meshless nodes. 


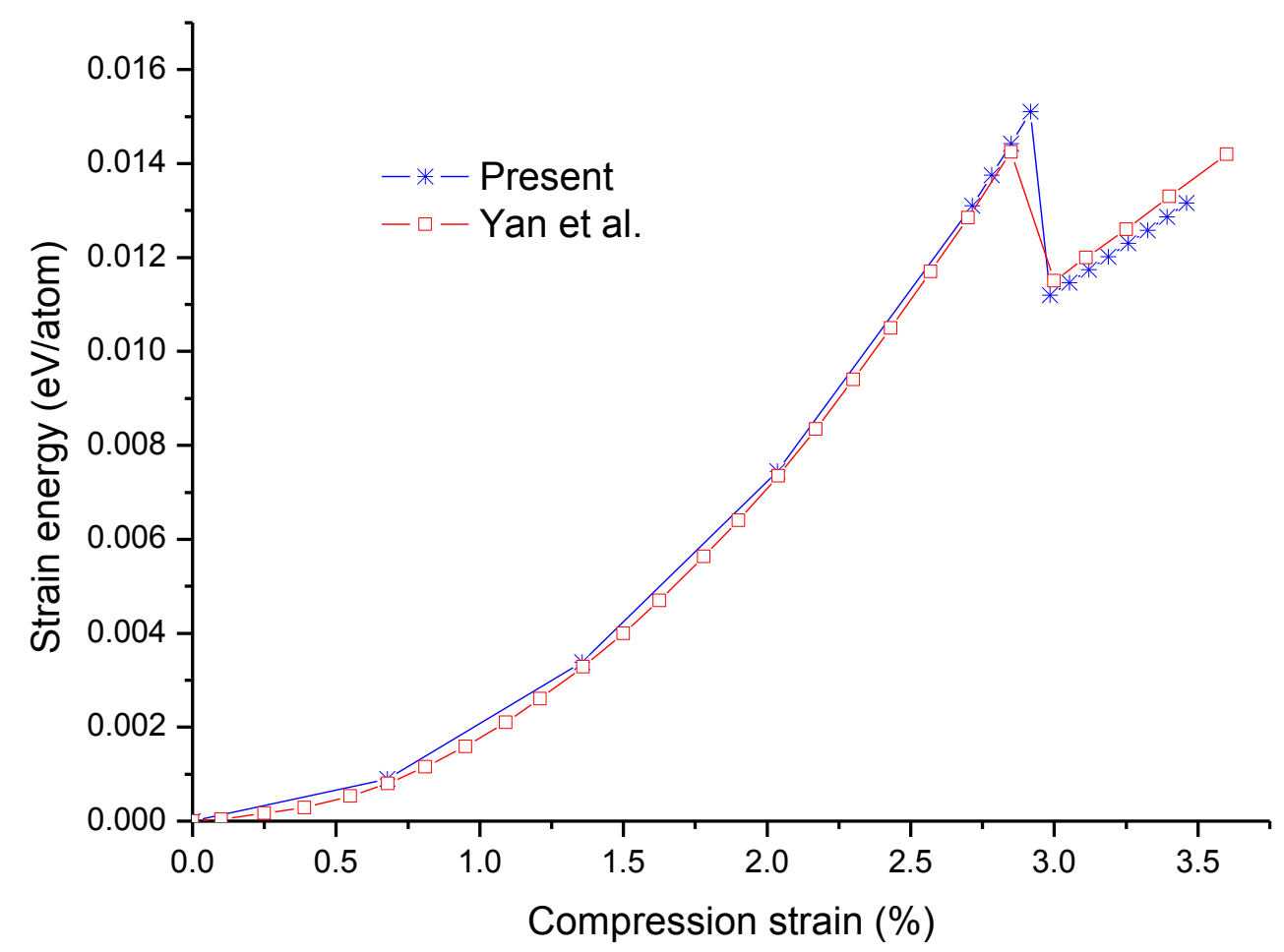

Fig. 7. The strain energy per atom versus the axial compression strain for the SWCNC with the geometrical parameters $\varphi=19.2^{\circ}, H_{c}=7.37 \mathrm{~nm}$ and $r_{c}=0.6 \mathrm{~nm}$. 


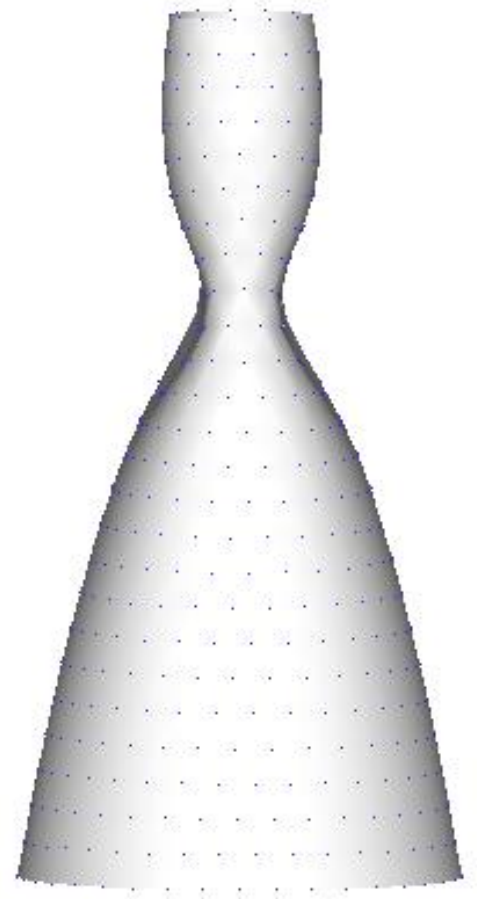

(a)

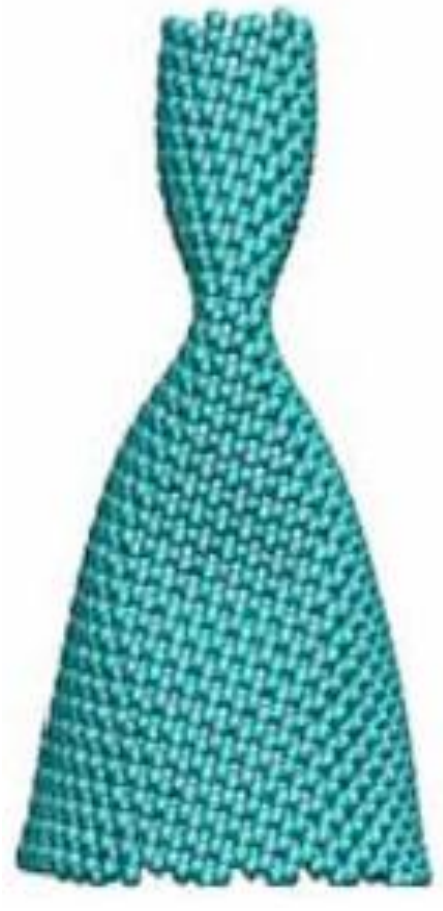

(b)

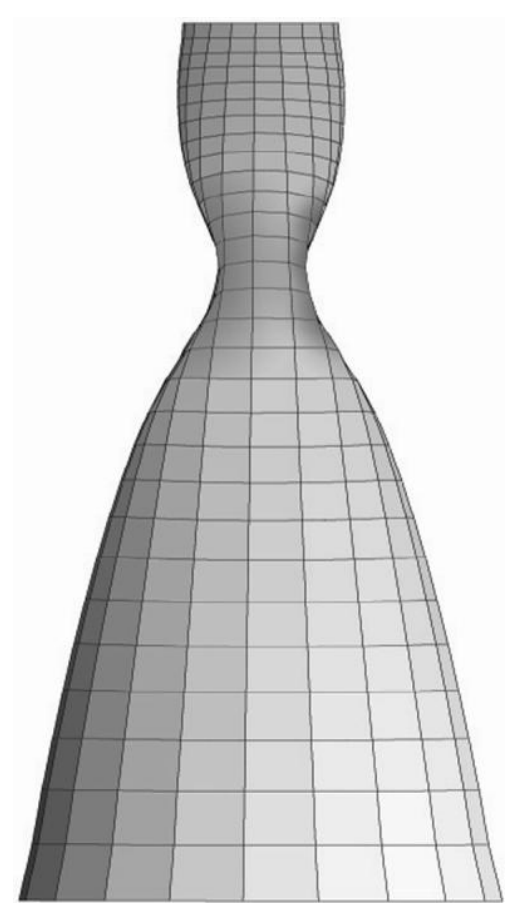

(c)

Fig. 8. The buckling patterns of SWCNCs by (a) the presented temperature-related QC model, (b) MD simulation [4] and (c) QC model [8], respectively. 


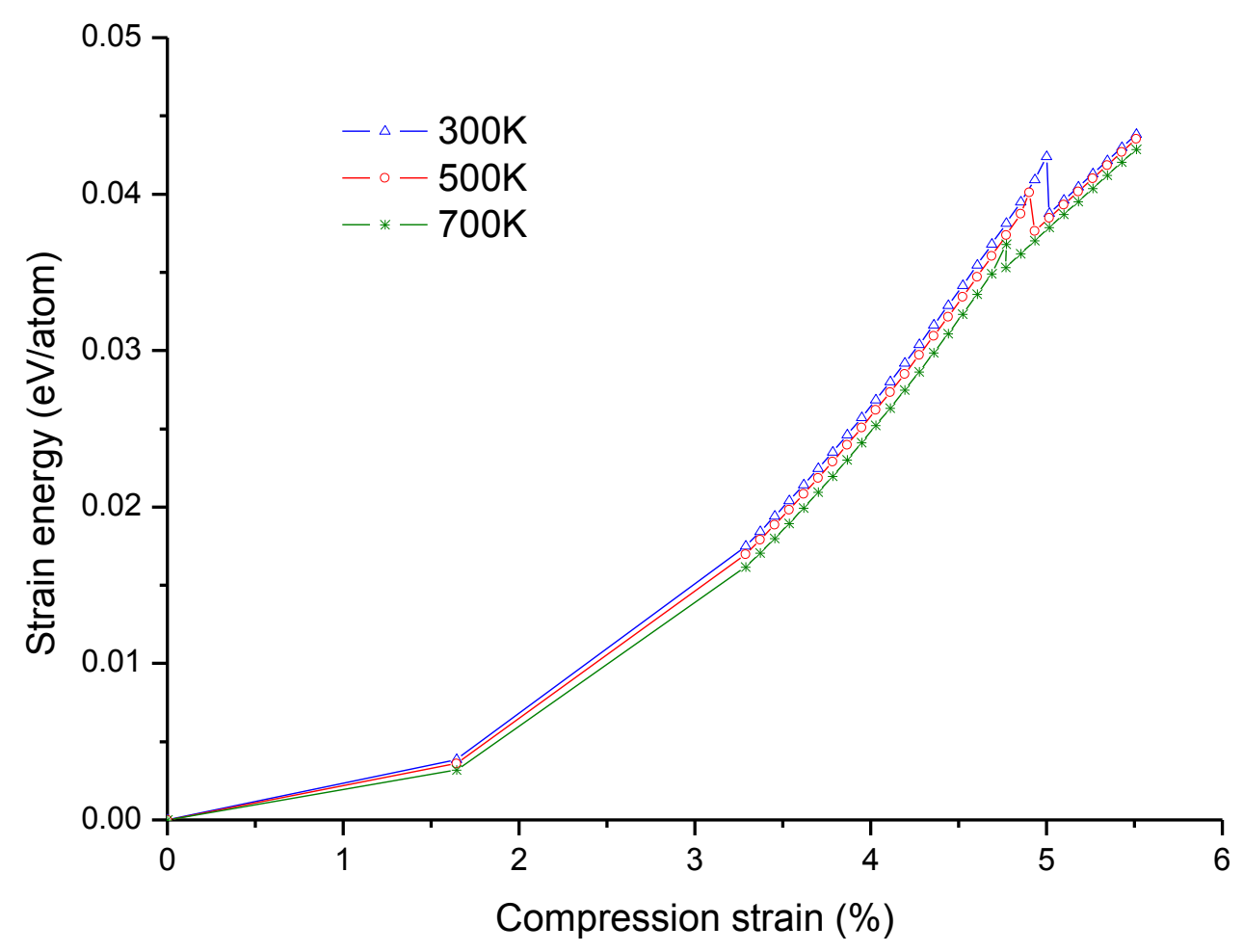

Fig. 9. The strain energy per atom versus axial compression strain of the SWCNC with $\varphi=19.2^{\circ}, H_{c}=3.04 \mathrm{~nm}$ and $r_{c}=0.34 \mathrm{~nm}$, respectively, at different temperatures. 


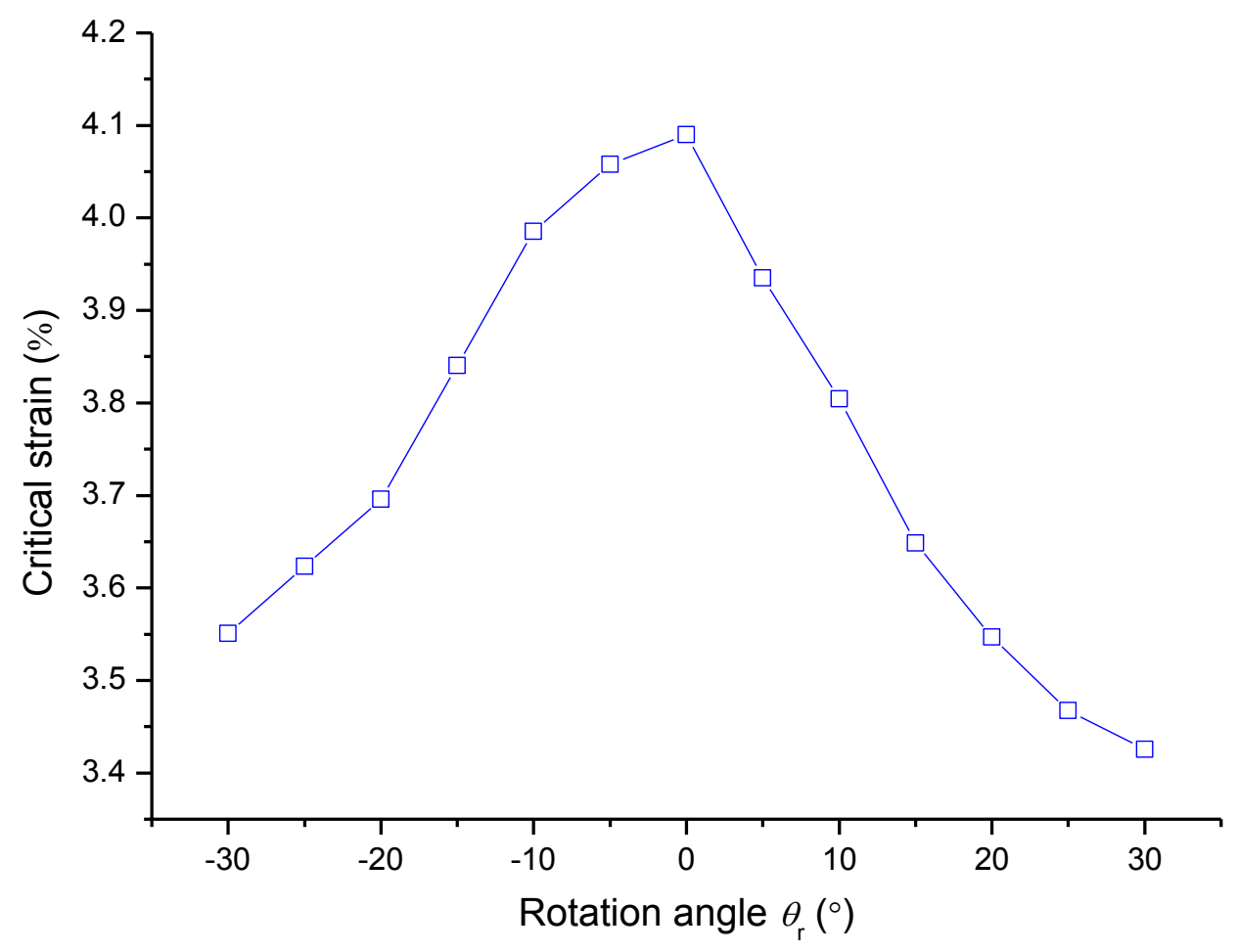

Fig. 10. The critical compression strain versus the rotation angle of cutting lines for $\operatorname{SWCNC}\left(\varphi=19.2^{\circ}, H_{c}=6.9 \mathrm{~nm}\right.$ and $\left.r_{c}=0.33 \mathrm{~nm}\right)$ at $300 \mathrm{~K}$. 


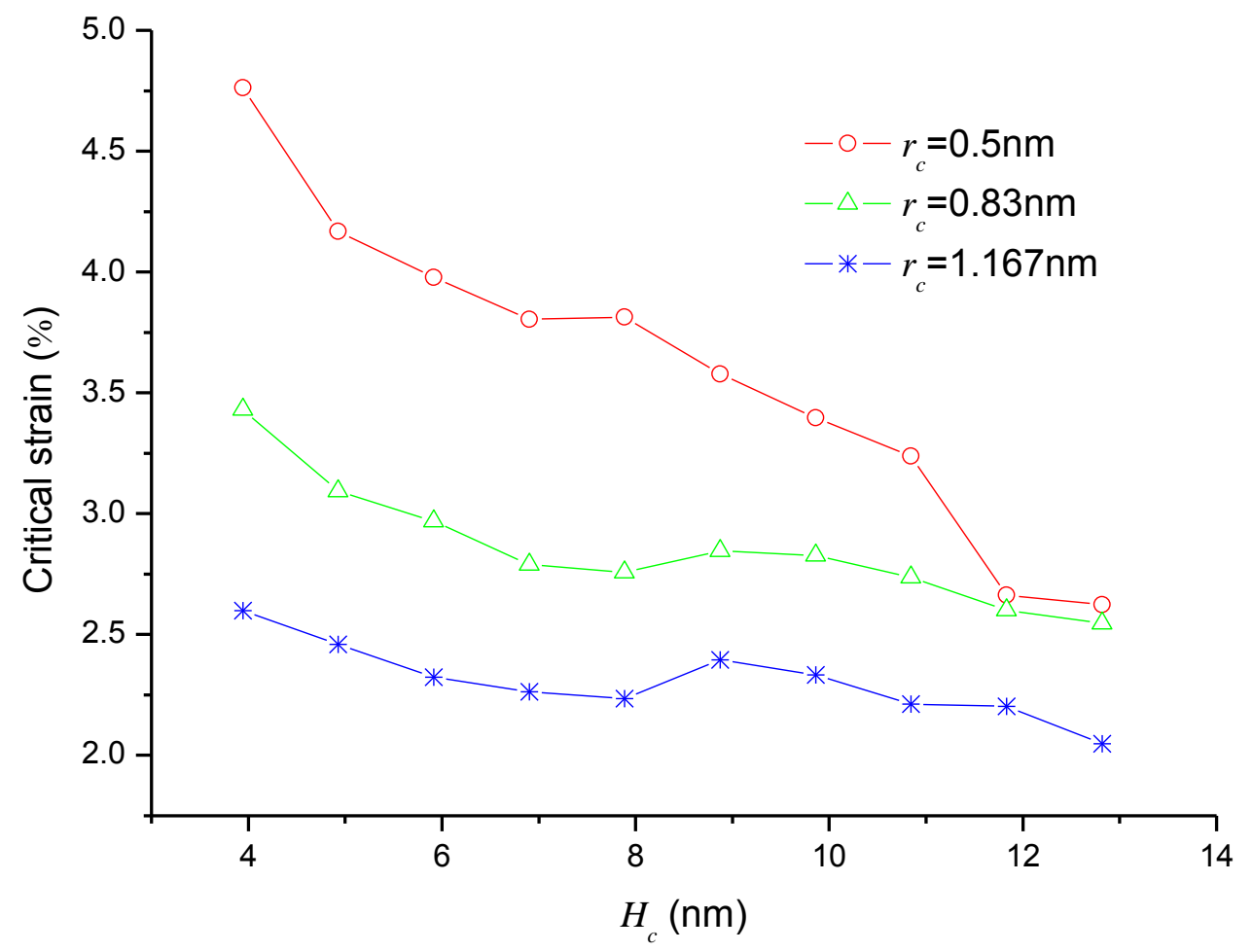

Fig. 11. The variation of the critical compression strain with respect to the height of SWCNCs with top radius of $0.5 \mathrm{~nm}, 0.83 \mathrm{~nm}$ and $1.167 \mathrm{~nm}$, respectively, at $300 \mathrm{~K}$. 


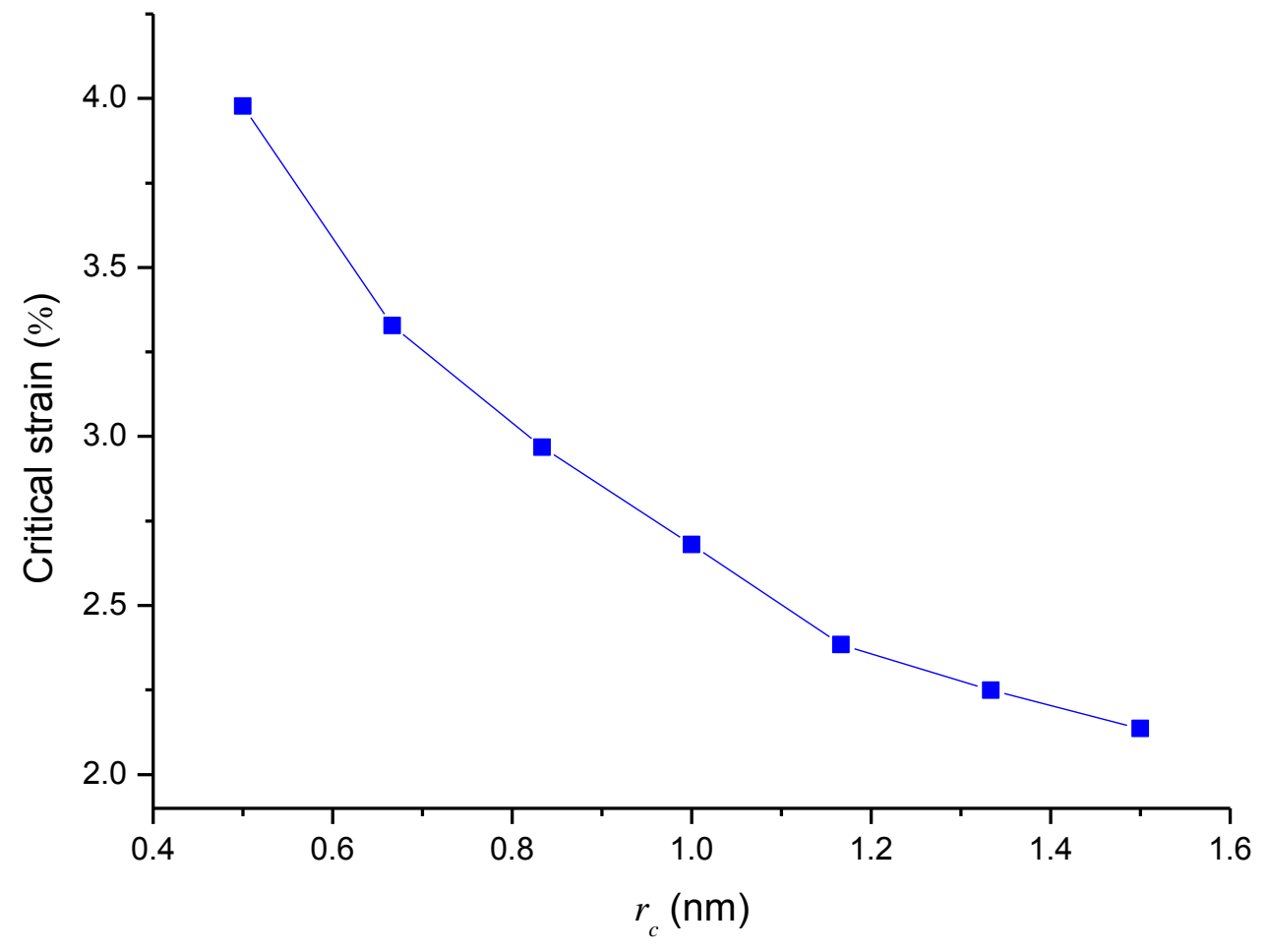

Fig. 12. The critical compression strain versus top radius for SWCNCs with height of $5.92 \mathrm{~nm}$ at $300 \mathrm{~K}$. 


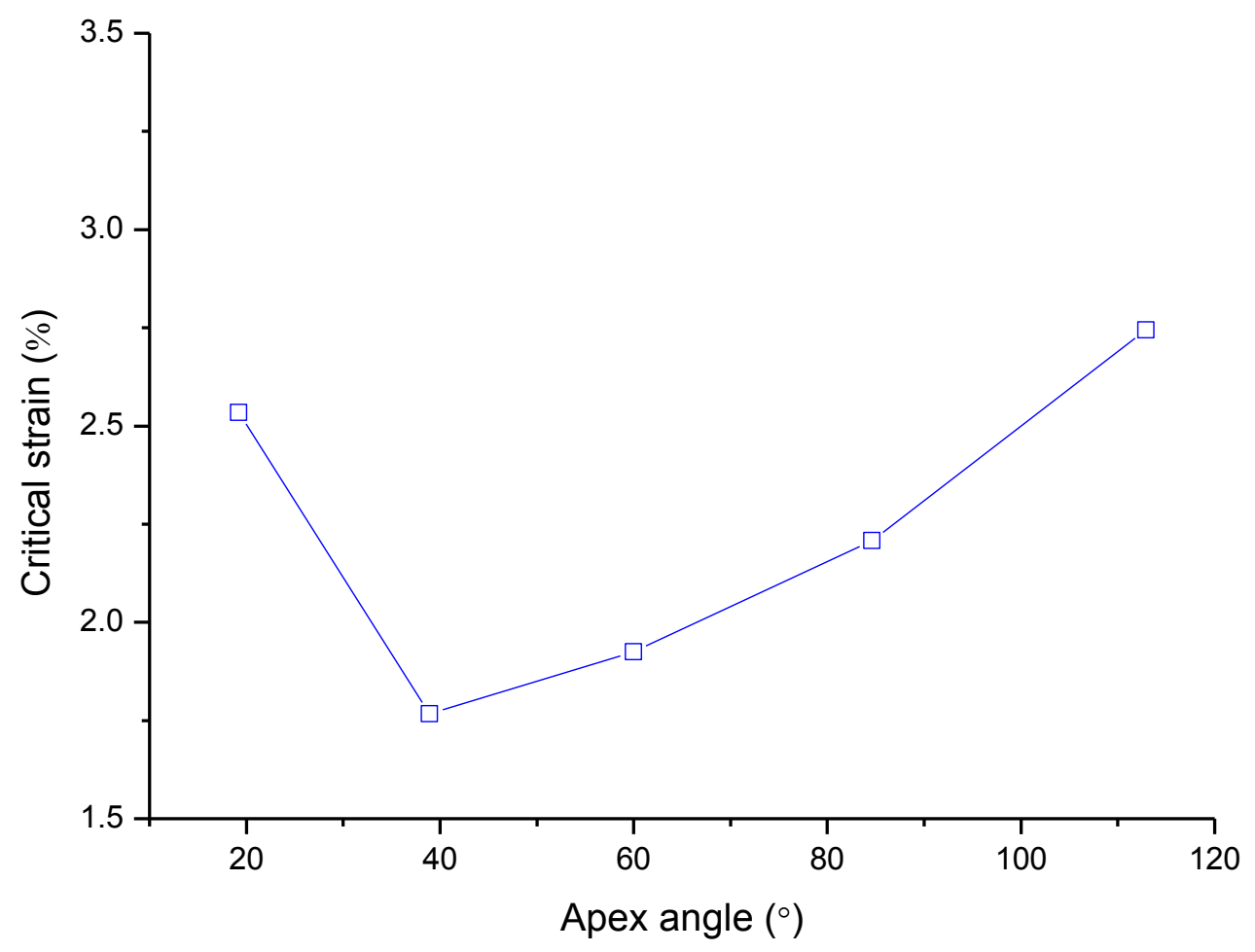

Fig. 13. The critical compression strain versus the apex angle for the SWCNCs with the same side edge length $6 \mathrm{~nm}$ and the top radius of $1 \mathrm{~nm}$ at $300 \mathrm{~K}$. 

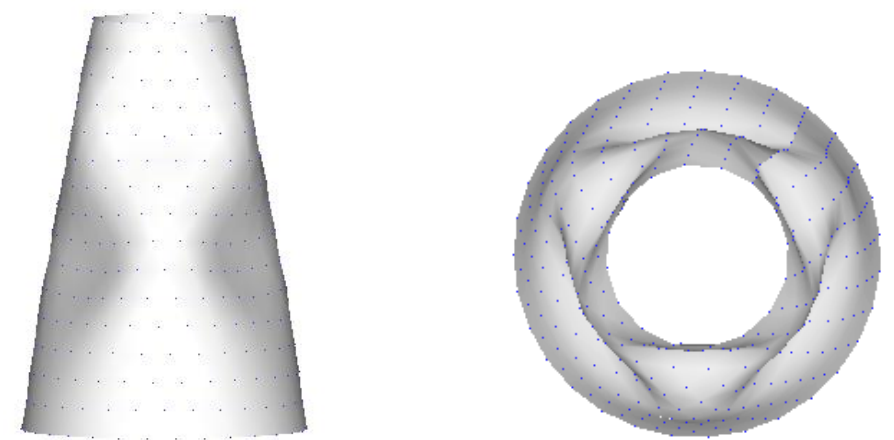

(a)
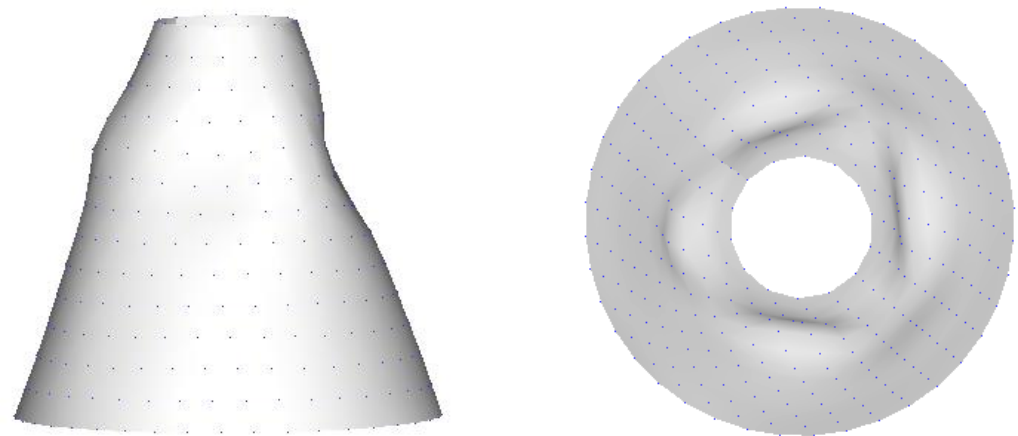

(b)
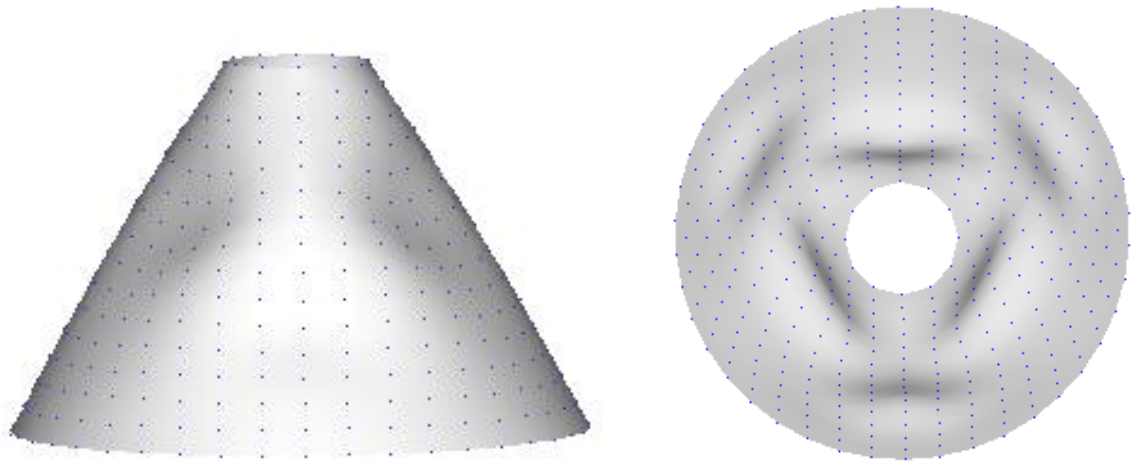

(c)

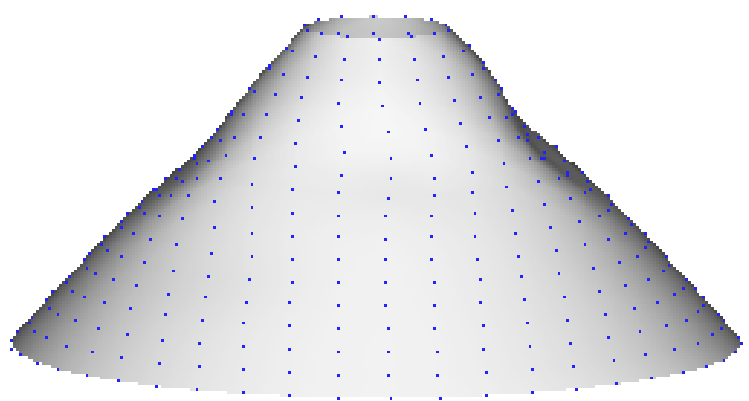

(d)

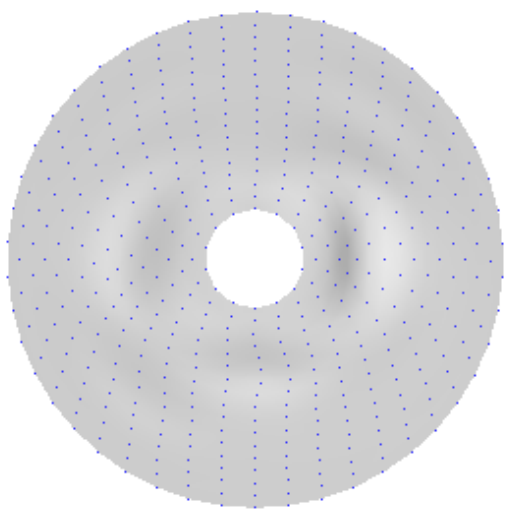



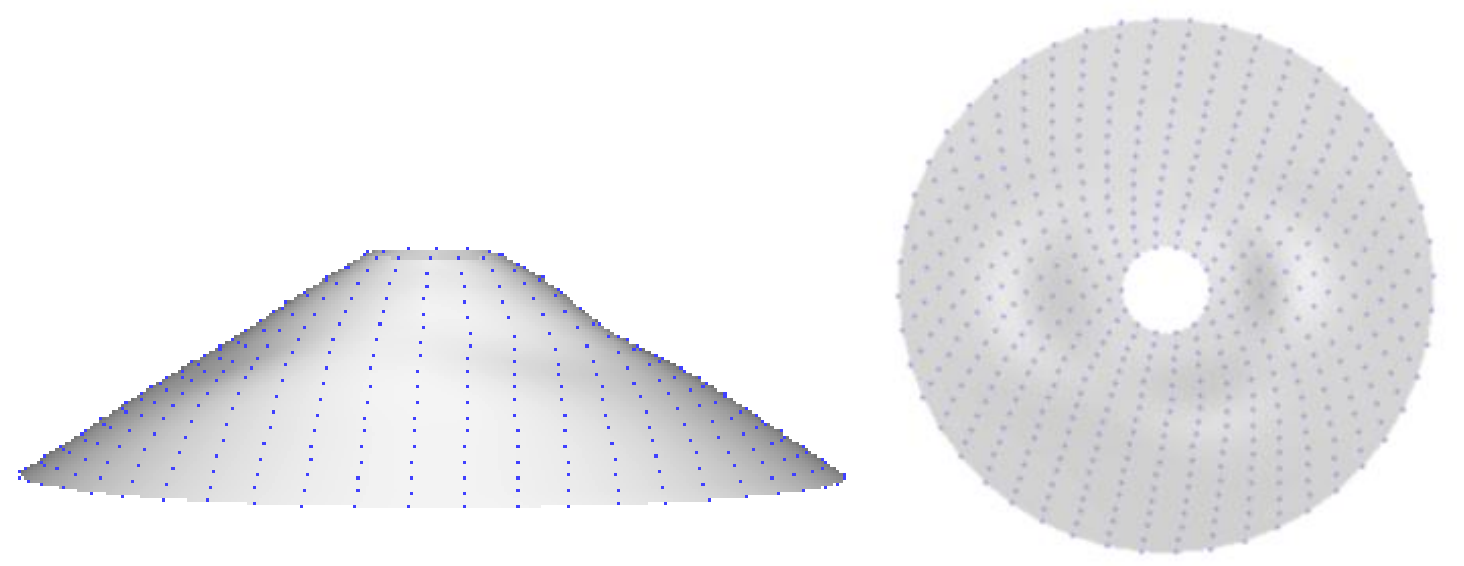

(e)

Fig. 14. The buckling patterns of the SWCNCs with the same side length $6 \mathrm{~nm}$ and top radius $1 \mathrm{~nm}$, the apex angles of the SWCNCs: (a) $19.2^{\circ}$, (b) $38.9^{\circ}$, (c) $60^{\circ}$, (d) $84.6^{\circ}$ and (e) $112.9^{\circ}$ 